\title{
Intranasal Immunization with Nontypeable Haemophilus influenzae Outer Membrane Vesicles Induces Cross- Protective Immunity in Mice
}

\author{
Sandro Roier ${ }^{1}$, Deborah R. Leitner ${ }^{1}$, Jeremy Iwashkiw ${ }^{2}$, Kristina Schild-Prüfert ${ }^{1}$, Mario F. Feldman ${ }^{2}$, \\ Georg Krohne ${ }^{3}$, Joachim Reidl ${ }^{1}$, Stefan Schild ${ }^{1 *}$
}

1 Institute of Molecular Biosciences, University of Graz, Graz, Austria, 2 Alberta Glycomics Centre, Department of Biological Sciences, University of Alberta, Edmonton, Alberta, Canada, 3 Division of Electron Microscopy, Biocenter of the University of Würzburg, Würzburg, Germany

\begin{abstract}
Haemophilus influenzae is a Gram-negative human-restricted bacterium that can act as a commensal and a pathogen of the respiratory tract. Especially nontypeable $H$. influenzae (NTHi) is a major threat to public health and is responsible for several infectious diseases in humans, such as pneumonia, sinusitis, and otitis media. Additionally, NTHi strains are highly associated with exacerbations in patients suffering from chronic obstructive pulmonary disease. Currently, there is no licensed vaccine against NTHi commercially available. Thus, this study investigated the utilization of outer membrane vesicles (OMVs) as a potential vaccine candidate against NTHi infections. We analyzed the immunogenic and protective properties of OMVs derived from various NTHi strains by means of nasopharyngeal immunization and colonization studies with BALB/c mice. The results presented herein demonstrate that an intranasal immunization with NTHi OMVs results in a robust and complex humoral and mucosal immune response. Immunoprecipitation revealed the most important immunogenic proteins, such as the heme utilization protein, protective surface antigen D15, heme binding protein A, and the outer membrane proteins P1, P2, P5 and P6. The induced immune response conferred not only protection against colonization with a homologous NTHi strain, which served as an OMV donor for the immunization mixtures, but also against a heterologous NTHi strain, whose OMVs were not part of the immunization mixtures. These findings indicate that OMVs derived from NTHi strains have a high potential to act as a vaccine against NTHi infections.
\end{abstract}

Citation: Roier S, Leitner DR, Iwashkiw J, Schild-Prüfert K, Feldman MF, et al. (2012) Intranasal Immunization with Nontypeable Haemophilus influenzae Outer Membrane Vesicles Induces Cross-Protective Immunity in Mice. PLoS ONE 7(8): e42664. doi:10.1371/journal.pone.0042664

Editor: Lisa A. Morici, Tulane University School of Medicine, United States of America

Received February 13, 2012; Accepted July 10, 2012; Published August 3, 2012

Copyright: (c) 2012 Roier et al. This is an open-access article distributed under the terms of the Creative Commons Attribution License, which permits unrestricted use, distribution, and reproduction in any medium, provided the original author and source are credited.

Funding: This work was supported by the Austrian Science Fund (FWF): grant W901-B12 (DK Molecular Enzymology) to SR and SS and grant I 662-B09 to JR. The authors acknowledge the Alberta Glycomics Centre for funding to MFF. MFF is a Canadian Institutes of Health Research (CIHR) new investigator and Alberta Heritage Foundation for Medical Research (AHFMR) scholar. The funders had no role in study design, data collection and analysis, decision to publish, or preparation of the manuscript.

Competing Interests: The authors have declared that no competing interests exist.

*E-mail: stefan.schild@uni-graz.at

\section{Introduction}

Haemophilus influenzae is a Gram-negative coccobacillus that commonly colonizes the human respiratory tract as a commensal or pathogen. This bacterium can be differentiated into typeable and nontypeable strains based on the presence or absence of a polysaccharide capsule. Encapsulated strains are divided into six capsular serotypes $(\mathrm{a}-\mathrm{f})$, with serotype $\mathrm{b}$ (Hib) being the most common associated with human disease. Infections caused by Hib strains range mainly from meningitis and acute epiglottitis to sepsis. In contrast, nonencapsulated and therefore nontypeable $H$. influenzae (NTHi) strains generally cause pneumonia, sinusitis, and otitis media $[1,2,3]$. Additionally, NTHi strains are one of the most common bacterial cause of exacerbations in patients suffering from chronic obstructive pulmonary disease (COPD) [4,5]. According to the latest WHO estimates, over 3 million people died of COPD in 2004 and it is predicted that COPD will become the third leading cause of death worldwide by 2030 [6].

Since the introduction of capsular polysaccharide conjugate vaccines against $\mathrm{Hib}$ in the late 1980s, invasive Hib diseases have been dramatically reduced in many developed countries [7]. In contrast, invasive diseases caused by NTHi infections have been steadily recognized since Hib vaccination began and have become the most frequent cause of an invasive $H$. influenzae disease in some regions $[8,9,10,11]$. Due to this altered epidemiology of invasive $H$. influenzae infections, particularly acute otitis media, and the increasing burden of COPD-related morbidity and mortality, there is a high demand for an effective NTHi vaccine. Besides the fact that NTHi strains have no conserved capsule, the key limitations for vaccine development are the high genetic heterogeneity of NTHi strains as well as the enormous antigenic variability of several surface-exposed antigens $[1,12,13]$. Therefore, vaccine development has focused on highly conserved structures of outer membrane proteins (OMPs), lipooligosaccharide (LOS), or pili. Among the most promising vaccine candidates are OMPs like $\mathrm{P} 2, \mathrm{P} 4, \mathrm{P}$, $\mathrm{P}$ 6, protein $\mathrm{D}$ and $\mathrm{E}$, since these antigens are highly immunogenic and represent abundant surface proteins in many NTHi isolates $[13,14,15,16,17,18]$. In addition, also LOS conjugate vaccines against NTHi have been investigated $[19,20]$. In order to be immunogenic the detoxified LOS has to be conjugated to a carrier, such as the tetanus toxoid, high-molecularweight proteins or P6 [20,21]. Recent studies indicate that even 
P6, which was believed to be one of the most conserved OMPs in NTHi, is not conserved in all NTHi strains and may not be surface exposed [22,23]. Thus, focusing on single antigens might not be the best approach for an effective NTHi vaccine. Instead, presenting a combination of multiple heterologous antigens to the immune system could increase the efficacy of a vaccine against heterologous NTHi strains. In this regard, outer membrane vesicles $(\mathrm{OMVs})$ could be considered as a new promising vaccine candidate.

OMVs are natural secretion products of Gram-negative bacteria. They are released when parts of the outer membrane $(\mathrm{OM})$ bulge and pinch off in the form of spherical and bilayered vesicles. These vesicles range in size from 10 to $300 \mathrm{~nm}$ in diameter and consist mainly of OM components, such as phospholipids, OMPs, and lipopolysaccharide (LPS) or LOS. Additionally, OMVs contain periplasmic components, which are trapped in the lumen of OMVs at the vesiculation process $[24,25,26,27]$. Due to the release from the OM, OMVs reflect the natural composition of the OM $[24,28,29,30]$. Therefore, OMVs carry multiple native bacterial antigens, which, combined with their multi-immunogenic and self-adjuvant properties, make them of particular interest for vaccine development [31]. An additional important feature is that the surface-exposed membrane antigens of OMVs maintain their physico-chemical stability [32,33]. For these reasons, the immunogenic and protective properties of OMVs have been tested and proven for several bacterial species, e. g. Vibrio cholerae, Salmonella typhimurium, Borrelia burgdorferi, Bordetella pertussis, and Porphyromonas gingivalis [32,34,35,36,37,38,39]. However, the greatest success has been achieved with OMVs derived from Neisseria meningitidis. So far, OMV vaccines are the only protective formulation against $\mathcal{N}$. meningitidis serogroup $\mathrm{B}$ and have been extensively used in several countries demonstrating both safety and efficacy $[33,40]$.

To date, all Gram-negative bacteria that have been investigated for secretion of vesicles are able to naturally release OMVs $[25,27]$. The release of OMVs by $H$. influenzae was first described for a nonencapsulated serotype d strain correlating with the loss of competence $[41,42]$. The presence of OMVs in NTHi cultures has been documented by two studies using electron microscopy $[16,43]$. A recent study identified 142 proteins present in OMVs derived from NTHi strain 86-028NP [44]. Consistent with OMV proteomes of other Gram-negative bacteria and the current models of OMV biogenesis mainly periplasmic proteins, OMPs or $\mathrm{OM}$ associated proteins have been elucidated.

In the present study the release of OMVs from several NTHi strains was analyzed by electron microscopy, protein profile comparisons and identification of abundant cargo proteins. Furthermore, we examined the capacity of OMVs derived from NTHi strains to act as a potential vaccine against NTHi infections by means of nasopharyngeal immunization and colonization studies with $\mathrm{BALB} / \mathrm{c}$ mice. Therefore, we investigated the humoral, mucosal, and protective immune responses against homologous and heterologous NTHi strains after intranasal immunization with NTHi OMVs.

\section{Materials and Methods}

\section{Ethics statement}

Female BALB/c mice (Charles River Laboratories) were used for all immunization experiments in strict accordance with the recommendations in the Guide for the Care and Use of Laboratory Animals of the National Institutes of Health, the national "Bundesgesetzblatt fuer die Republik Oesterreich". The corresponding animal protocol (39/158 ex 2000/10), has been approved by the Austrian Federal Ministry of Science and Research Ref. II/10b and the Committee on the Ethics of Animal Experiments of the University of Graz. Mice were housed with food and water ad libitum and monitored under the care of full-time staff and in accordance with the rules of the Institute of Molecular Biosciences at the University of Graz. All animals were acclimated for 1 week before any procedures were carried out and were 9 weeks old at the start of the immunization.

\section{Bacterial strains and growth conditions}

The NTHi strains 1479, 2019, 3198, 5657, 7502, and 9274 were kindly provided by Michael A. Apicella, University of Iowa, USA. Additionally, the spontaneous streptomycin-resistant $\left(\mathrm{Sm}^{\mathrm{r}}\right)$ derivative AC53 of $V$. cholerae strain E7946 was used [45]. All NTHi strains are clinical isolates from the sputum or middle ear fluid of human patients and have been described previously [21,46]. Spontaneous $\mathrm{Sm}^{\mathrm{r}}$ derivatives 1479-R, 2019-R, 3198-R, 7502-R, and 9274-R of the respective NTHi strains as well as 5657 were used in all experiments. $\mathrm{Sm}^{\mathrm{r}}$ derivatives allowed the positive selection throughout the study including the challenge experiment and were generated by plating overnight cultures of the respective strains on brain heart infusion (BHI) agar supplemented with streptomycin. After $48 \mathrm{~h}, \mathrm{Sm}^{\mathrm{r}}$ colonies were recovered, purified, and compared with their respective donor wild-type strains for their OM and OMV protein profiles as well as for their growth kinetics. In all cases, no obvious differences were observed (data not shown).

Bacteria were grown at $37^{\circ} \mathrm{C}$ with aeration in $\mathrm{LB}$ broth or on $\mathrm{LB}$ agar in the case of $V$. cholerae as well as in BHI broth or on BHI agar supplemented with NAD and hemin-solution (stock-solution containing a mixture of hemin, L-histidine, and triethanolamine) in the case of NTHi. When appropriate, streptomycin was added. Supplements were used in the following final concentrations: NAD, $10 \mu \mathrm{g} / \mathrm{ml}$; hemin, $20 \mu \mathrm{g} / \mathrm{ml}$; L-histidine, $20 \mu \mathrm{g} / \mathrm{ml}$; triethanolamine, $0,08 \%$; and streptomycin, $100 \mu \mathrm{g} / \mathrm{ml}$.

\section{Multilocus sequence typing (MLST)}

MLST analysis of the NTHi isolates was performed as described in the MLST database (http://haemophilus.mlst.net) [47]. The sequences of seven genes for each isolate were submitted to the international MLST database to obtain the allelic profile of each sample as well as the sequence type (ST) of each isolate. In case of the $m d h$ locus of NTHi strain 1479, a new allelic number and a new MLST sequence type were assigned by a curator of the international database. The MLST results have been added to the database and are available online (http://haemophilus.mlst.net/).

\section{Preparation of OMPs and whole-cell lysates (WCL)}

Proteins of the OM were prepared according to Carlone et al. [48]. Briefly, cells from $10 \mathrm{ml}$ of the respective NTHi overnight culture were harvested by centrifugation $\left(3,200 \times \mathrm{g}, 10 \mathrm{~min}, 4^{\circ} \mathrm{C}\right)$. The cell pellet was washed once in HEPES buffer $(10 \mathrm{mM}$, $\mathrm{pH} 7.4$ ) and resuspended in $1 \mathrm{ml}$ HEPES buffer $(10 \mathrm{mM}, \mathrm{pH} 7.4)$ with protease inhibitor (Roche, Complete EDTA-free protease inhibitor cocktail, 1 tablet per $50 \mathrm{ml}$ ). Cells were disrupted by sonification (six bursts, 10 sec each) using a Branson Ultrasonics sonifier S-250A. Unbroken cells were removed by centrifugation $\left(15,600 \times \mathrm{g}, 2 \mathrm{~min}, 4^{\circ} \mathrm{C}\right)$. The supernatant containing the OMPs was transferred into a new tube and centrifuged again $(15,600 \times \mathrm{g}$, $30 \mathrm{~min}, 4^{\circ} \mathrm{C}$ ). The membrane pellet was resuspended in $0.4 \mathrm{ml}$ HEPES buffer (10 mM, pH 7.4). To solubilize the cytoplasmic membrane, $0.4 \mathrm{ml}$ HEPES buffer $(10 \mathrm{mM}$, pH 7.4$)$ with $2 \%$ sarcosyl was added and incubated at room temperature (RT) for 30 min with intermittent mixing. After centrifugation $(15,600 \times \mathrm{g}$, 
$30 \mathrm{~min}, 4^{\circ} \mathrm{C}$ ), the pellet containing the OMPs was washed once with $0.5 \mathrm{ml}$ HEPES buffer $(10 \mathrm{mM}, \mathrm{pH} 7.4)$ and finally resuspended in $50 \mu \mathrm{l}$ HEPES buffer $(10 \mathrm{mM}$, pH 7.4). Purified OMPs were stored at $-20^{\circ} \mathrm{C}$.

WCL were prepared by sonification similar to the preparation of OMPs as described above. After removal of unbroken cells by centrifugation, the remaining supernatant served as WCL. Purified WCL were stored at $-20^{\circ} \mathrm{C}$.

The protein concentrations of OMP and WCL preparations were determined by photometric measurements of the absorbances at $260 \mathrm{~nm}$ and $280 \mathrm{~nm}$ using a Beckman Coulter DU730 spectrophotometer in combination with a TrayCell (Hellma) and the Warburg-Christian equation.

\section{Preparation of OMVs}

$V$. cholerae OMVs were isolated as previously published [34]. NTHi OMVs were isolated accordingly, except for the following modifications: $500 \mathrm{ml} \mathrm{BHI}$ broth was inoculated with $5 \mathrm{ml}$ of the respective NTHi overnight culture and grown to late exponential phase for $13 \mathrm{~h}$. Bacterial cells were pelleted by two subsequent centrifugation steps $\left(6,400 \times \mathrm{g}, 10 \mathrm{~min}, 4^{\circ} \mathrm{C}\right.$ and $16,000 \times \mathrm{g}$, $6 \mathrm{~min}, 4^{\circ} \mathrm{C}$ ). The supernatant containing the OMVs was consecutively filtered through $0.45 \mu \mathrm{m}$ and $0.2 \mu \mathrm{m}$ pore size filters to ensure complete removal of remaining bacteria. $0.2 \mathrm{ml}$ of the filtrate was plated on a BHI agar plate and incubated $48 \mathrm{~h}$ to confirm the absence of viable bacteria. In all preparations, no colonies were observed. The filtrate was stored at $4^{\circ} \mathrm{C}$ and within the next two days OMVs were pelleted from the filtrate by ultracentrifugation $\left(144,000 \times \mathrm{g}, 4 \mathrm{~h}, 4^{\circ} \mathrm{C}\right)$ using a Beckman Coulter Optima L-100 XP ultracentrifuge and a SW 32 Ti rotor. The OMV pellet was resuspended in approximately $150 \mu \mathrm{l}$ phosphate-buffered saline (PBS, $\mathrm{pH} 7.4$ ) and stored at $-70^{\circ} \mathrm{C}$. The protein concentrations of the OMV preparations were determined as described above and adjusted to $2.5 \mu \mathrm{g} / \mu \mathrm{l}$ using PBS (pH 7.4).

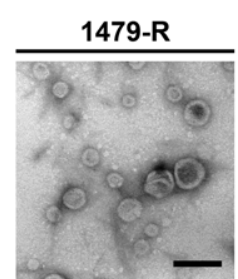

5657

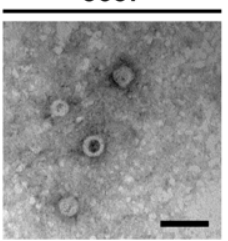

2019-R

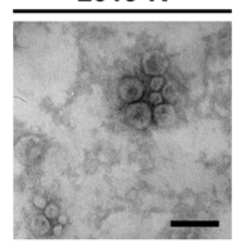

7502-R

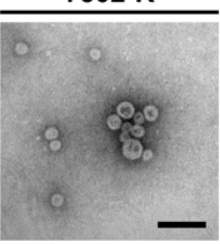

3198-R

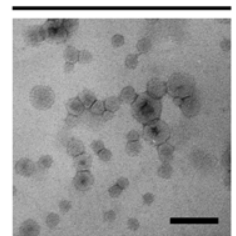

9274-R

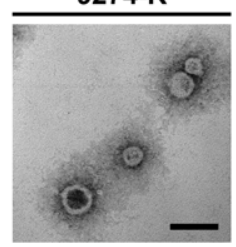

Figure 1. OMV and OM protein profiles of heterologous NTHi strains. Protein profile comparisons of OMV and OM preparations from the heterologous NTHi strains (A) 9274-R, 2019-R, and 1479-R, as well as (B) 7502-R, 5657, and 3198-R are shown. Samples (approx. $4 \mu \mathrm{g}$ protein each) were separated by SDS-PAGE $(12,6 \%$ gels) and protein bands were visualized according to Kang et al. [59]. Lines to the left indicate the molecular masses of the protein standards in $\mathrm{kDa}$.

doi:10.1371/journal.pone.0042664.g001

\section{Immunization protocol and experimental setup}

For the immunization with OMVs derived from various NTHi strains or $V$. cholerae, mice were divided into different immunization groups. These groups received either immunization mixture 1 (IM-1) consisting of OMVs derived solely from NTHi 2019-R (Table 1) via the intranasal or intraperitoneal (i.p. IM-1) administration route, immunization mixture 2 (IM-2) consisting of equally mixed OMVs derived from NTHi 2019-R, 9274-R, and 1479-R (Table 1) via the intranasal administration route, or OMVs derived from $V$. cholerae AC53 via the intranasal administration route (i.n. Vch-OMV). NTHi strain 2019-R was chosen as the sole donor for OMVs used in IM-1, because it was extensively used in several in vivo studies, it is the prototype strain

Table 1. Experimental setup of the immunization mixtures and NTHi strains used in this study with respect to their relevance for the immune systems of the immunized mice.

\begin{tabular}{|c|c|c|c|c|c|}
\hline \multirow[b]{2}{*}{$\begin{array}{l}\text { Immunization } \\
\text { mixture (IM) }\end{array}$} & \multirow[b]{2}{*}{$\begin{array}{l}\text { OMV donor strain } \\
\text { for the IM }\end{array}$} & \multicolumn{4}{|c|}{ Relevance for the immune systems of immunized mice } \\
\hline & & $\begin{array}{l}\text { Known }^{a} \text { LOS (LOS } \\
\text { group }^{b} \text { ) }\end{array}$ & $\begin{array}{l}\text { Known surface } \\
\text { proteins (OMP } \\
\text { subtype? }\end{array}$ & $\begin{array}{l}\text { Unknown }{ }^{a} \text { LOS (LOS } \\
\text { group) }\end{array}$ & $\begin{array}{l}\text { Unknown surface } \\
\text { proteins (OMP subtype) }\end{array}$ \\
\hline \multirow[t]{5}{*}{ IM-1 } & 2019- $\mathrm{R}^{d}$ & 2019-R (II) & 2019-R (2) & 1479-R (I) & 1479-R (1) \\
\hline & & & & $\underline{3198-R(I I I)}$ & $3198-R(8)$ \\
\hline & & & & 9274-R (III) & 9274-R (n.d. $\left.{ }^{e}\right)$ \\
\hline & & & & 5657 (IV) & $5657(7)$ \\
\hline & & & & $7502-\mathrm{R}(\mathrm{V})$ & $7502-\mathrm{R}(3)$ \\
\hline \multirow[t]{4}{*}{ IM-2 } & 1479-R & $1479-\mathrm{R}(\mathrm{I})$ & $1479-\mathrm{R}(1)$ & 5657 (IV) & $5657(7)$ \\
\hline & 2019-R & 2019-R (II) & $2019-R(2)$ & $7502-\mathrm{R}(\mathrm{V})$ & $7502-\mathrm{R}(3)$ \\
\hline & 9274-R & 9274-R (III) & 9274-R (n.d.) & & $\underline{3198-R(8)}$ \\
\hline & & 3198-R (III) & & & \\
\hline
\end{tabular}

${ }^{a}$ Known and unknown refer to the presence or absence of the particular LOS or surface proteins in the immunization mixtures, respectively.

bLOS group classification as previously published $[19,49]$.

'According to the OMP subtype classification developed by Murphy et al. [46] and the heterologous protein profiles shown in Fig. 1.

${ }^{\mathrm{d}}$ Strains underlined were used for challenge.

${ }^{\mathrm{e}}$ Not determined by Murphy et al. [46].

doi:10.1371/journal.pone.0042664.t001 
for LOS group II, and its LOS structure has been identified $[19,49,50,51,52,53,54]$. In addition to 2019-R OMVs, also OMVs derived from NTHi strains $9274-\mathrm{R}$ and $1479-\mathrm{R}$ were used in IM-2 to increase the antigen complexity of the immunization mixture. These strains were chosen, because they have been classified into different LOS groups (type I, II and III) and demonstrate a high OM heterogeneity $[19,46,49]$. The LOS structure of NTHi strain 9274-R was identified [55] and differs significantly from NTHi strain 2019-R [52]. Besides the LOS structure, the diversity of these strains is also indicated by their heterogeneous OMV protein profiles and allocation into different ST (Fig. 1A and Table 2). Antibody titers in serum to OMVs were monitored at four time points before (day 0), during (day 14 and 28), and after (day 39) the immunization period by using an indirect ELISA and plates coated with OMVs derived from various NTHi strains serving as antigen. ELISA plates coated with 2019-R OMVs were used to determine the immunoglobulin responses against a homologous strain, whose surface antigens were presented (known) to the immune systems of mice immunized with either IM-1 or IM-2. In contrast, OMVs derived from the heterologous NTHi strains 3198-R, 5657, and 7502-R [19,46,49] were neither present in IM1 nor IM-2. The strains 5657 and 7502-R are the prototype strains for the LOS groups IV and V, respectively. Thus, ELISA plates coated with OMVs derived from these strains were used to determine the immune responses against heterologous strains, whose surface antigens were not presented (unknown) to the immune systems of the immunized mice. In contrast, strain 3198$\mathrm{R}$ exhibits $\mathrm{OM}$ heterogeneity in the protein profile, but belongs to the LOS group III like the strain 9274-R, which was used as one donor for OMVs provided in IM-2. Therefore, ELISA plates coated with OMVs derived from strain 3198-R reflect a heterologous strain, whose surface protein antigens were unknown, while a similar LOS structure has been presented to the immune systems of the mice immunized with IM-2. In the case of mice immunized with IM-1, surface protein and LOS antigens of 3198-R were unknown to the immune system.

For all immunization experiments a nonvaccinated control group of sham immunized mice with PBS $(\mathrm{pH} 7.4)$ were housed in parallel with vaccinated mice for the duration of the experiment. No adjuvant was used for the entire study. To avoid potential effects by coprophagia mice of each immunization groups as well as the control mice were kept in separated cages. Mice were intranasally $(25 \mu \mathrm{g}$ OMVs in $10 \mu \mathrm{l} \mathrm{PBS}, 5 \mu \mathrm{l}$ per nostril) or intraperitoneally $(2 \mu \mathrm{g}$ OMVs in $100 \mu \mathrm{l}$ PBS $)$ immunized at days 0,14 , and 28 . This immunization doses were based on previously published immunization studies using $\mathrm{OMVs}$ derived from $V$. cholerae and $\mathcal{N}$. meningitidis [34,35,56,57]. Mice were briefly anesthetized by inhalation of $2.5 \%$ isoflurane gas prior to all immunizations. None of the animals died throughout the immunization study and no significant differences in consumption of food and water was detected between cages harbouring vaccinated mice and control mice. Overall two independent rounds of immunization for each immunization group were performed with at least 3 mice per round and different batches of OMV preparations, respectively. Comparison of the data from the independent immunization rounds revealed no differences in the induced immune response or protection for the respective immunization groups.

\section{Collection and preparation of blood and stool samples}

Blood samples were collected by lateral tail vein nick at day 0 , 14, and 28, as well as by cardiac puncture at day 39. Additionally, three to five freshly voided fecal pellets per mouse were collected at day 39. Samples were processed as described previously [34,35]. Serum samples and extracted Igs from the fecal pellets were stored at $-70^{\circ} \mathrm{C}$.

\section{Challenge with NTHi}

All vaccinated and nonvaccinated control mice were challenged with NTHi at day 38 for $24 \mathrm{~h}$. Mice were inoculated with approximately $5 \times 10^{5}$ CFU of either NTHi 2019-R or NTHi 3198-R. To prepare the inoculum, the respective NTHi strains were grown in BHI broth to an optical density at $490 \mathrm{~nm}\left(\mathrm{OD}_{490}\right)$ of 1. Cells were harvested by centrifugation $(2,300 \times \mathrm{g}, 5 \mathrm{~min}$, RT), resuspended in PBS (pH 7.4), and adjusted to an $\mathrm{OD}_{490}$ of 1.1 (equivalent to approximately $1 \times 10^{9} \mathrm{CFU} / \mathrm{ml}$ ). Subsequently, 1:10 dilutions in PBS ( $\mathrm{pH} 7.4$ ) were prepared. The first 1:10 dilution was mixed 1:1 with PBS $(\mathrm{pH} 7.4)$ to obtain the inoculum (approximately $5 \times 10^{7} \mathrm{CFU} / \mathrm{ml}$ ). In parallel appropriate dilutions were plated on BHI plates supplemented with streptomycin and incubated at $37^{\circ} \mathrm{C}$ for two days to determine the $\mathrm{CFU} / \mathrm{ml}$ of the inoculum by back-calculating to the original suspension. Prior to challenge, mice were briefly anesthetized by inhalation of $2.5 \%$ isoflurane gas. Then mice were intranasally inoculated with approximately $5 \times 10^{5} \mathrm{CFU}$ using $10 \mu \mathrm{l}(5 \mu \mathrm{l}$ per nostril $)$ of the inoculum. After $24 \mathrm{~h}$, the mice were sacrificed and the nasopharynx from each mouse was removed by dissection. The nasopharynx was mechanically homogenized in BHI broth with $15 \%$ glycerol and appropriate 1:10 dilutions were plated on BHI plates supplemented with streptomycin. After incubation at $37^{\circ} \mathrm{C}$ for two days, the colonization rates in $\mathrm{CFU} /$ nasopharynx were determined by back-calculation to the original volume of the homogenized nasopharynges.

Table 2. Comparative MLST analysis of the NTHi strains used in this study.

\begin{tabular}{|c|c|c|c|c|c|c|c|c|}
\hline \multirow[b]{2}{*}{ Strain } & \multicolumn{7}{|c|}{ Allelic profile ${ }^{a}$} & \multirow[b]{2}{*}{$\mathrm{ST}^{a}$} \\
\hline & adk & $\operatorname{atpG}$ & $f r d B$ & fuck & $m d h$ & $p g i$ & $\operatorname{rec} A$ & \\
\hline 1479 & 68 & 43 & 55 & 11 & 216 & 58 & 18 & 1003 \\
\hline 2019 & 28 & 14 & 1 & 1 & 62 & 13 & 1 & 321 \\
\hline 3198 & 33 & 8 & 16 & 16 & 49 & 2 & 3 & 107 \\
\hline 5657 & 1 & 8 & 1 & 14 & 22 & 14 & 13 & 145 \\
\hline 7502 & 14 & 7 & 13 & 1 & 136 & 83 & 83 & 380 \\
\hline 9274 & 1 & 1 & 1 & 14 & 59 & 14 & 5 & 243 \\
\hline
\end{tabular}

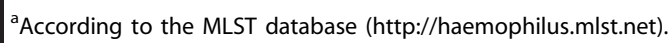

doi:10.1371/journal.pone.0042664.t002 


\section{Quantitation of antibodies}

Immunoglobulin A (IgA), IgG1, and IgM antibodies as well as half-maximum total Ig titers (IgA, IgG, and IgM) to OMVs were quantified by indirect enzyme-linked immunosorbent assay (ELISA) using 96-well ELISA microplates (BD Falcon) essentially as described previously [34,35]. ELISAs were performed by using appropriate OMVs $(5 \mu \mathrm{g} / \mathrm{ml}$ in PBS, $\mathrm{pH} 7.4)$ as coating antigens as well as appropriate Ig isotype standards (BD Biosciences) and horseradish peroxidase-conjugated goat anti-mouse secondary antibodies (Southern Biotech). For detection, the TMB peroxidase substrate reagent set (BioLegend) was used according to the manufacturer's instructions. Optical densities were read at $450 \mathrm{~nm}$ with a FLUOstar Omega microplate reader (BMG Labtech). Antibody titers were calculated using values from appropriate dilutions of the test samples and a log-log regression calculated from at least four dilutions of the isotype standards. Halfmaximum total $\mathrm{Ig}$ titers were calculated by plotting the $\log$ of the reciprocal dilutions of mouse sera against the resulting absorbances to create sigmoidal curves, which were used to determine the reciprocals that gave half of the theoretical maximum optical density.

\section{SDS-PAGE and immunoblot analysis}

In order to analyze the protein content of the OM, WCL, and OMVs, proteins were separated by sodium dodecyl sulfatepolyacrylamide gel electrophoresis (SDS-PAGE) [58] in combination with 12.6\% gels using the Prestained Protein Marker Broad Range (New England Biolabs) as a molecular mass standard. Protein bands were visualized according to Kang et al. [59].

Immunoblot analysis was performed as described previously [34]. Chemiluminescence detection was performed by using the Immun-Star ${ }^{\mathrm{TM}}$ WesternC ${ }^{\mathrm{TM}}$ Kit (Bio-Rad Laboratories) and subsequent exposure in a ChemiDoc XRS system (Bio-Rad Laboratories) in combination with Quantity One software (BioRad Laboratories).

\section{Immunoprecipitation}

Immunoprecipitation was performed by using the Dynabeads ${ }^{\circledR}$ Protein G Immunoprecipitation Kit (Invitrogen) according to the manufacturer's manual. To avoid mouse-specific variations, sera collected on day 39 from all mice immunized with IM-1 were pooled and $16 \mu \mathrm{l}$ of this mixture was used for binding of the antibodies to the beads. $16 \mu \mathrm{l}$ of pooled serum collected on day 39 from the nonvaccinated control mice served as a negative control. $100 \mu \mathrm{l}$ of an OMP preparation $(2 \mu \mathrm{g} / \mu \mathrm{l})$ from strain 2019-R l was used as antigen. Proteins in the immunoprecipitations were separated by SDS-PAGE and analyzed by mass spectrometry.

\section{MALDI TOF-TOF MS and MS/MS analysis}

Protein bands from the OMV and immunoprecipitation samples were excised and in-gel digested using sequencing grade modified trypsin (Promega) [60]. Peptide fragments were eluted from the gel piece, desalted using $\mathrm{ZipTipC}_{18}$ (Millipore) according to the supplier protocol, resuspended in $0.1 \%$ formic acid, spotted onto a Bruker Daltonics MTP AnchorChip ${ }^{\text {TM }}$ 800/384 target and air dried. $0.42 \mu \mathrm{L}$ of $\alpha$-cyano-4-hydroxy cinnamic acid matrix solution (CHCA) was spotted on top and air dried. The matrix solution was prepared by diluting $36 \mu \mathrm{L}$ of saturated matrix solution in $0.1 \%$ TFA in 90:10 ACN: $\mathrm{H}_{2} \mathrm{O}$ to $800 \mu \mathrm{L}$ final volume using $0.1 \%$ TFA in 85:15 ACN: $\mathrm{H}_{2} \mathrm{O}$, containing $1 \mathrm{mM}$ ammonium phosphate. Mass spectra were obtained in the positive reflectron mode of ionization using a Bruker Daltonics (Bremen, Germany) UltrafleXtreme MALDI TOF/TOF mass spectrome- ter. The MS and MS/MS spectra were obtained in an automated mode of operation. For MS/MS analysis the CID (collisioninduced dissociation) gas was turned off. The FlexAnalysis, WARP-LG, ProteinScape software packages provided by the manufacturer and an in house MASCOT server were used for analysis of the mass spectra.

\section{Transmission electron microscopy}

For OMV visualization by electron microscopy, vesicles were purified as described above and adjusted to a final concentration of $2.5 \mu \mathrm{g} / \mu \mathrm{l}$ using PBS (pH 7.4). Samples were fixed in $0.5 \%$ glutaraldehyde $(10 \mathrm{mM}$ cacodylate $\mathrm{pH} 7.2,10 \mathrm{mM} \mathrm{KCl}, 0.5 \mathrm{mM}$ $\mathrm{MgCl}_{2}$ ) for 5 min and subsequently diluted 1:100 in $\mathrm{H}_{2} \mathrm{O} .12 \mu \mathrm{l}$ of the dilutions were allowed to adsorb onto glow-discharged 300mesh carbon coated copper grid for $1 \mathrm{~min}$ followed by negative staining with aqueous uranyl acetate $(0.5 \%)$. The micrographs were recorded at an accelerating voltage of $80 \mathrm{kV}$ using a EM10 electron microscope (Zeiss, Oberkochen, Germany). The photographic negatives were digitalized by scanning and processed using Adobe Photoshop (Adobe Systems Incorporated).

\section{Statistical analysis}

Data were analyzed using the Mann-Whitney U test or a Kruskal-Wallis test followed by post hoc Dunn's multiple comparisons. Differences were considered significant at $P$ values of $<0.05$. For all statistical analyses, GraphPad Prism version 4.0a for Mac OS X (GraphPad Software) was used.

\section{Results}

\section{NTHi strains release OMVs}

To investigate whether the NTHi strains used in this study are able to release OMVs, we adapted an established method to isolate $V$. cholerae OMVs derived from culture supernatants [34]. Six heterologous NTHi strains were tested for OMV production. These included the five prototype NTHi strains 1479-R (type I), 2019-R (type II), 3198-R (type III), 5657 (type IV) and 7502-R (type $\mathrm{V}$ ) based on the LOS typing system developed by Campagnari et al. [49]. Additionally, we used the NTHi strain 9274-R (type III), which has been used for various LOS vaccination studies $[19,20,21,61,62,63]$. Phylogenetic relationship of the NTHI strains was characterized by MLST analysis to prove the diversity of the NTHi strains. In the case of NTHI strains 2019 and 9274, the MLST data was already available in the respective database (http://haemophilus.mlst.net/). We identified the allelic profile and sequence types (ST) for the NTHi strains 1479, 3198, 5657 and 7502, which subsequently allowed a comparison of all six NTHi strains (Table 2). The isolates exhibited high variation in their allelic profiles. Consequently all strains are allocated into different ST. The best match was obtained for NTHi strains 5657 and 9274 with only 3 identical alleles out of 7 , while all other comparisons revealed less then 3 identical alleles. Thus, the six strains used in this study are highly heterologous.

Cell-free vesicle samples of these heterologous NTHi strains were isolated from late log-phase cultures using filtration and ultracentrifugation steps (see Materials and Methods for details). Purified vesicles were analyzed by transmission electron microscopy (Fig. 2). All samples contained multiple spherical vesicles, the majority of which ranged between 20 and $80 \mathrm{~nm}$ in size. The protein patterns of the purified OMVs were analyzed and compared with the respective OM preparations by SDS-PAGE in combination with Kang's staining method (Fig. 1). All strains tested revealed similar protein profiles of the respective OMV and OM samples, indicating that the abundant proteins of the $\mathrm{OM}$ are 
also present in the derived OMVs. Some protein bands are overor underrepresented in the OMV preparations compared to the OM preparations, but such enrichments and exclusions of proteins in OMVs have been described before for other bacteria $[64,65,66,67,68]$. The surface protein heterogeneity of these NTHi strains is highlighted by their heterogeneous OMV or OMP profiles, especially by the variable migration patterns of the two most abundant protein bands located between 25 and $46 \mathrm{kDa}$. Thus, all strains used in this study reflect the typical variation in the OMP patterns, which is a hallmark of NTHi strains [46,69,70,71,72,73,74].

To exclude variability within OMV preparations of a respective NTHi strain, we compared the protein profiles from OMV samples isolated from independent cultures of each NTHi strain. Representative examples are provided in Fig. 3 showing three independent preparations of OMVs derived from NTHi strain 2019-R (Fig. 3A) and 3198-R (Fig. 3B), which are the most important samples used in this study. For both strains, similar patterns and intensities of the protein bands in these OMV preparations derived from three independent cultures can be observed. Such low variations in independent OMV preparations were also observed for all other strains used in this study (data not shown). This indicates that at least the protein composition of the OMVs is highly reproducibility and a constant quality can be achieved in independent OMV preparations.

In order to characterize the protein cargo of the vesicles, the most intensive protein bands of the OMVs derived from NTHi strain 2019-R were excised and subjected to tryptic digestion and mass spectrometry. The analysis identified eight proteins of the NTHi 2019-R OMVs, with the OMPs P2 and P5 including their smaller sized degradation products being the most abundant ones (Fig. 3A). Additionally, OMPs or OM associated proteins like the heme utilization protein, the IgA-specific serine endoprotease, the protective surface antigen D15, a putative lipoprotein, the OMP $\mathrm{P} 1$ and the opacity protein have been identified. Consistent with our data, these proteins were also found in the proteome of OMVs derived from NTHi strain 86-028NP [44].

In summary, our data confirms that NTHi strains in fact release OMVs during growth and that these OMVs can be reproducibly isolated by a simple protocol based on filtration and ultracentrifugation.
Immunization with NTHi OMVs induces humoral and mucosal immune responses

Mice were intranasally immunized with NTHi OMVs at day 0 , 14, and 28 using the two immunization mixtures IM-1 and IM-2, respectively. Table 1 provides a schematic overview of the experimental setup and a detailed description of the design as well as the composition of the immunization mixtures can be found in the Materials and Methods ("Immunization protocol and experimental setup"). Briefly, IM-1 contained only OMVs derived from NTHi strain 2019-R1, whereas IM-2 was composed of equally mixed OMVs derived from NTHi strain 2019-R 9274-R and $1479-\mathrm{R}$ to increase the antigen complexity of the immunization mixture. Antibody titers in serum to OMVs were monitored by ELISA using OMVs derived from various NTHi strains as coating antigen. ELISA plates coated with 2019-R OMVs were used to determine the immunoglobulin responses against a homologous strain, whose surface antigens were presented (known) to the immune systems of mice immunized with either IM-1 or IM-2. In contrast, OMVs derived from the heterologous NTHi strains 3198-R, 5657, and 7502-R were neither present in IM-1 nor IM-2. Thus, ELISA plates coated with OMVs derived from these strains were used to determine the immune responses against heterologous strains, whose surface antigens were not presented (unknown) to the immune systems of the immunized mice.

The temporal IgM, IgA, and IgG1 responses to OMVs derived from 2019-R or 3198-R are shown in Fig. 4 and Fig. 5, respectively. The IgM, IgA, and IgG1 titers of the nonvaccinated control group were determined only for day 0 and 39, since in general no significant increases were observed within this period ( $P>0.05$; Mann-Whitney U test). At day 0, the median isotypespecific antibody titers to OMVs derived from 2019-R or 3198-R were relatively low and showed no significant differences between the three groups $(P>0.05$; Kruskal-Wallis test and post hoc Dunn's multiple comparisons). It should be noted that at day 0 the median IgM titers of both immunization groups and the nonvaccinated control group were at least 20-fold increased compared to the respective median IgA and IgG1 titers (Fig. 4 and 5). The median IgM titers peaked at day 14 or 28 in both immunization groups followed by slight to moderate declines, most likely due to isotype switching (Fig. 4A and 5A). In contrast, the median IgA and IgG1 antibody titers to 2019-R OMVs (Fig. 4B and C) or 3198-R

A
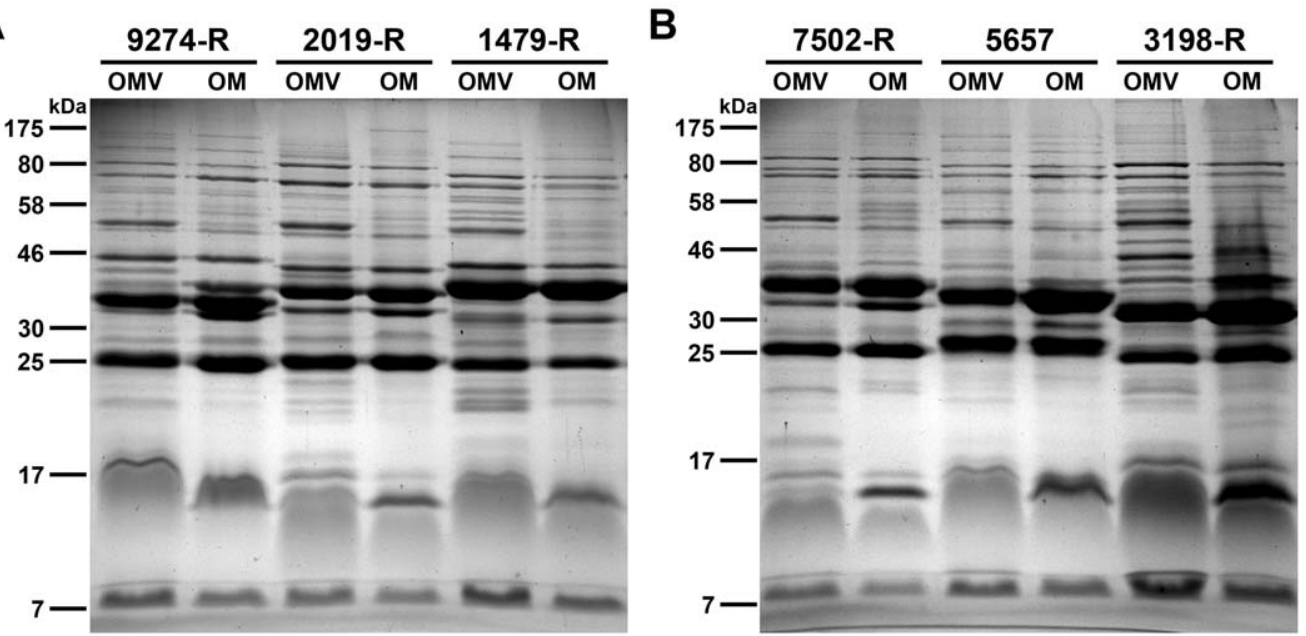

Figure 2. OMV visualization by electron microscopy. Transmission electron microscopy of purified OMVs derived from NTHi strain 1479-R, 2019-R, 3198-R, 5657, 7502-R and 9274-R negatively stained with uranyl acetate. The scale bars represent $100 \mathrm{~nm}$. doi:10.1371/journal.pone.0042664.g002 
A

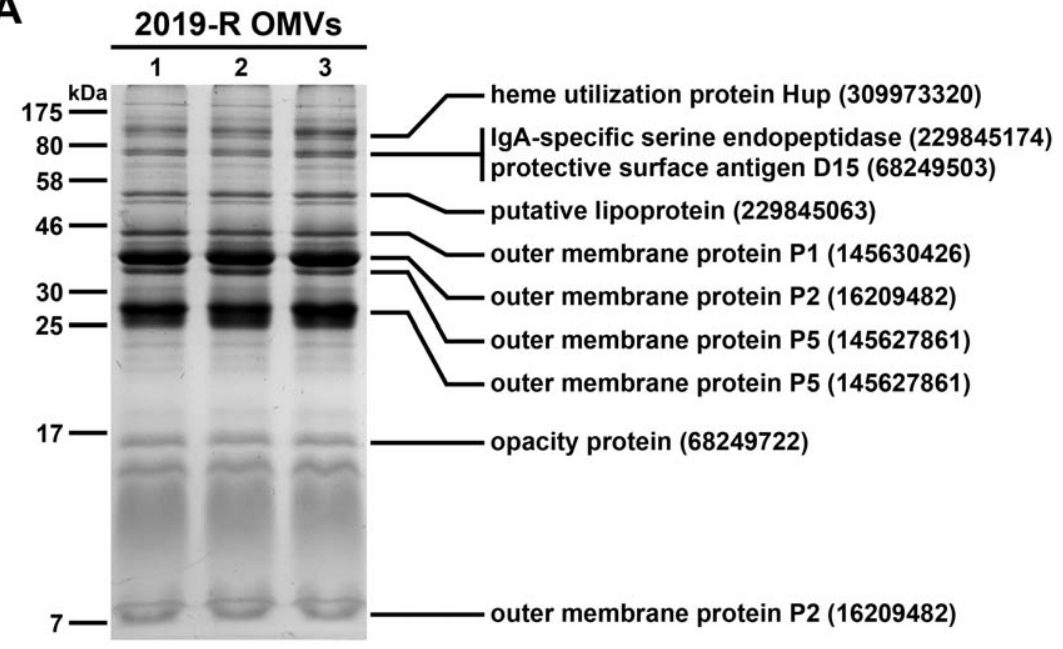

B

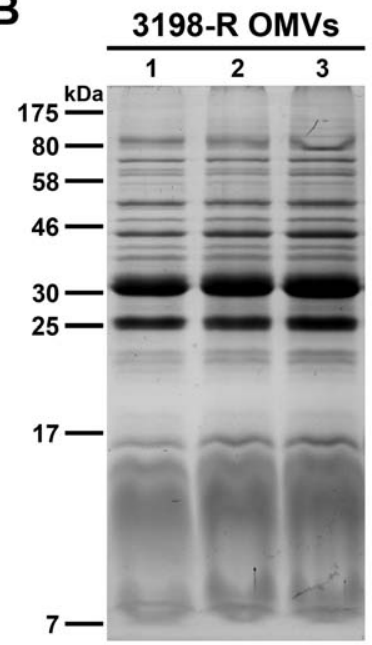

Figure 3. OMV protein profiles of independent preparations. Shown are protein profile comparisons of three independent OMV preparations from the NTHi strains 2019-R (A) and 3198-R (B). Samples (approx. $4 \mu \mathrm{g}$ protein each) were separated by SDS-PAGE (12,6\% gels) and protein bands were visualized according to Kang et al. [59]. Lines to the left indicate the molecular masses of the protein standards in kDa. Proteins identified by mass spectrometry from NTHi 2019-R OMVs are indicated with their respective position on the gel, protein identities and accession numbers on the right.

doi:10.1371/journal.pone.0042664.g003

OMVs (Fig. 5B and $\mathrm{C}$ ) of both immunization groups increased during the immunization period.

We also determined the half-maximum total Ig titers in sera collected at day 39 from mice of both immunization groups as well as from nonvaccinated control mice to homologous and heterologous OMVs derived from the NTHi strains 2019-R, 3198-R, 5657, and 7502-R (Fig. 6). The half-maximum total Ig titers allowed us to simultaneously detect $\operatorname{IgM}$, as well as abundant subclasses of $\operatorname{IgG}$ and $\operatorname{IgA}$ and therefore served as important immunological reference values to determine the total immunogenicities of the immunization mixtures. In all cases, the halfmaximum total Ig titers of mice immunized with either IM-1 or IM-2 were significantly higher than those of the nonvaccinated control mice $(P<0.05$; Kruskal-Wallis test and post hoc Dunn's multiple comparisons). Depending on the OMV type coated on the ELISA plates, the median half-maximum total Ig titers of the immunization groups were 30- to 500-fold higher for 5657 and 3198-R (Fig. 6C and B), respectively, if compared to the nonvaccinated control group.

In addition to the humoral immune responses, we also determined the induced mucosal immune responses by measuring the secretory IgA titers in fecal pellet extracts collected at day 39 from mice of both immunization groups as well as from nonvaccinated control mice to $\mathrm{OMV}$ s derived from either the homologous NTHi strain 2019-R (Fig. 7A) or the heterologous NTHi strain 3198-R (Fig. 7B). Both immunization groups showed significantly higher median secretory IgA titers to 2019-R and 3198-R OMVs compared to the nonvaccinated control group (Fig. 7; $P<0.05$; Kruskal-Wallis test and post hoc Dunn's multiple comparisons).

\section{OMVs contain numerous proteins that can serve as antigens}

To test the specificity of the antibody response, immunoblot analyses using OMV, OM, and WCL preparations derived from the NTHi strains 2019-R, 3198-R, 5657 and 7502-R as antigens were performed. To analyze the IgG reactivity, immunoblots were incubated with sera collected at day 39 from one mouse either immunized with IM-1 (Fig. 8A) or IM-2 (Fig. 8B) as well as from a nonvaccinated control mouse (Fig. 8C). No bands were detected on immunoblots using sera from nonvaccinated control mice (Fig. 8C). In contrast, multiple bands in the OMV, OM, and WCL protein profiles of the homologous NTHi strain 2019-R and even the heterologous NTHi strains 3198-R, 5657 and 7502-R were detected by using sera from the immunized mice (Fig. 8A and B). This clearly demonstrates that the OMVs used in IM-1 and IM-2 contain numerous proteins that can serve as antigens. In general, the most reactive bands are located at approx. 50, 35, 25, and $15 \mathrm{kDa}$.

To identify some immunogenic proteins of NTHi OMVs, we performed immunoprecipitation analyses using sera collected at day 39 from IM-1 immunized mice as antibodies and OM preparations of strain NTHi strain 2019-R as target antigens. Sera from nonvaccinated control mice served as a negative control. The corresponding SDS-PAGE profiles of the immunoprecipitates are shown in Fig. 9. Besides the heavy chain mouse immunoglobulin migrating at approximately $50 \mathrm{kDa}$, all the other protein bands appeared to be more intensive in the immunoprecipiation using sera from immunized mice compared to the nonvaccinated control mice. The faint bands present in the control could be explained by intrinsic level of natural IgM antibodies to NTHi, which also bind to the protein $\mathrm{G}$ used for the immunoprecipitation. Their presence is indicated by the relatively high initial IgM titers (Fig. 4 and 5), which have not been detected in immunizations studies analyzing OMVs derived from $V$. cholerae as a vaccine candidate [34,35]. Additionally, we cannot exclude that the OM preparations used in the immunoprecipitation contained protein complexes, which were not completely dissociated and could have been pulled down as aggregates. That is why we only focused on the most intensive protein bands of the immunoprecipiation using sera from immunized mice. These bands were excised and subjected to mass spectrometry. This analysis elucidated the seven immunogenic proteins that are indicated with their respective position in the gel provided in Fig. 9. Amongst others, the protective surface antigen D15 as well as the OMPs P2, P5 and P6 were identified to 
A

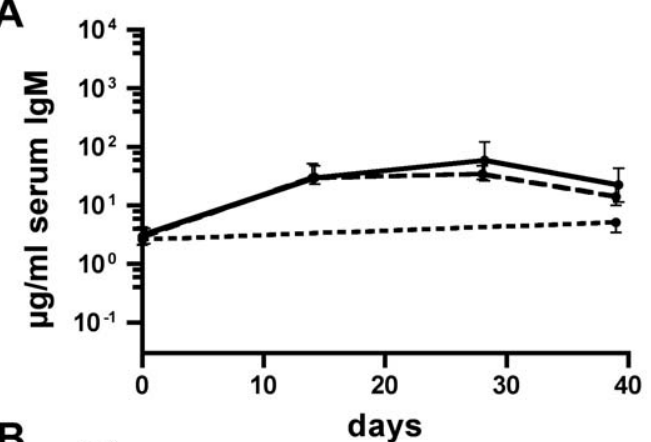

B

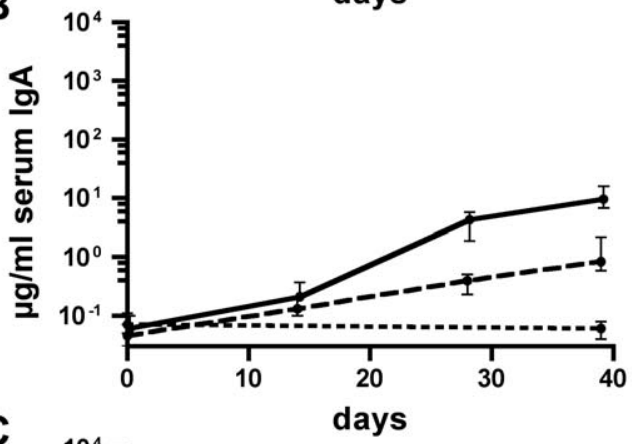

C

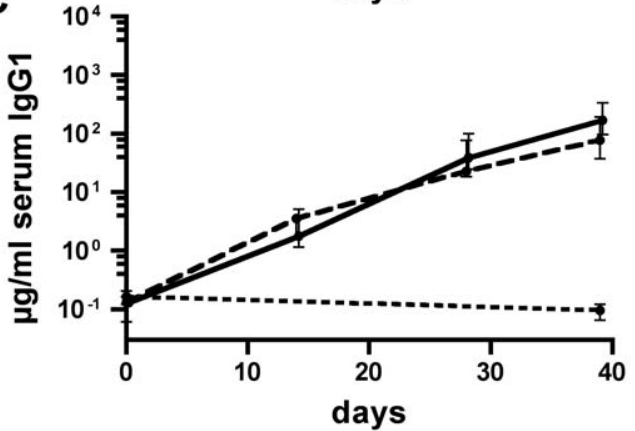

Figure 4. Temporal immune responses to OMVs derived from NTHi strain 2019-R. Shown are the median titers over time of $\operatorname{lgM}(A)$, IgA (B), and IgG1 (C) antibodies to 2019-R OMVs in sera from mice intranasally immunized with either IM-1 (solid line) or IM-2 (dashed line) as well as in sera from nonvaccinated control mice (dotted line) $(n=20$ for each group). The error bars indicate the interquartile range of each data set for each time point.

doi:10.1371/journal.pone.0042664.g004

be important antigens of this NTHi vaccine candidate based on OMVs.

\section{Immunization with NTHi OMVs induces cross-protective immunity}

In order to investigate whether the induced immune responses were protective against NTHi colonization of the mouse nasopharynx, we challenged all vaccinated mice, which were intranasally immunized with either IM-1 or IM-2, as well as all nonvaccinated control mice with the NTHi strains 2019-R or 3198-R. Therefore, all groups were divided into two subgroups and challenged intranasally with either the homologous NTHi strain 2019-R or the heterologous NTHi strain 3198-R at day 38. To determine the right infection doses of these NTHi strains, preliminary experiments were performed demonstrating that an infection dose of approximately $10^{4} \mathrm{CFU} /$ mouse is just sufficient for a stable colonization of both strains over $24 \mathrm{~h}$ (data not shown). To ensure a stable colonization, we challenged the mice with a 50-
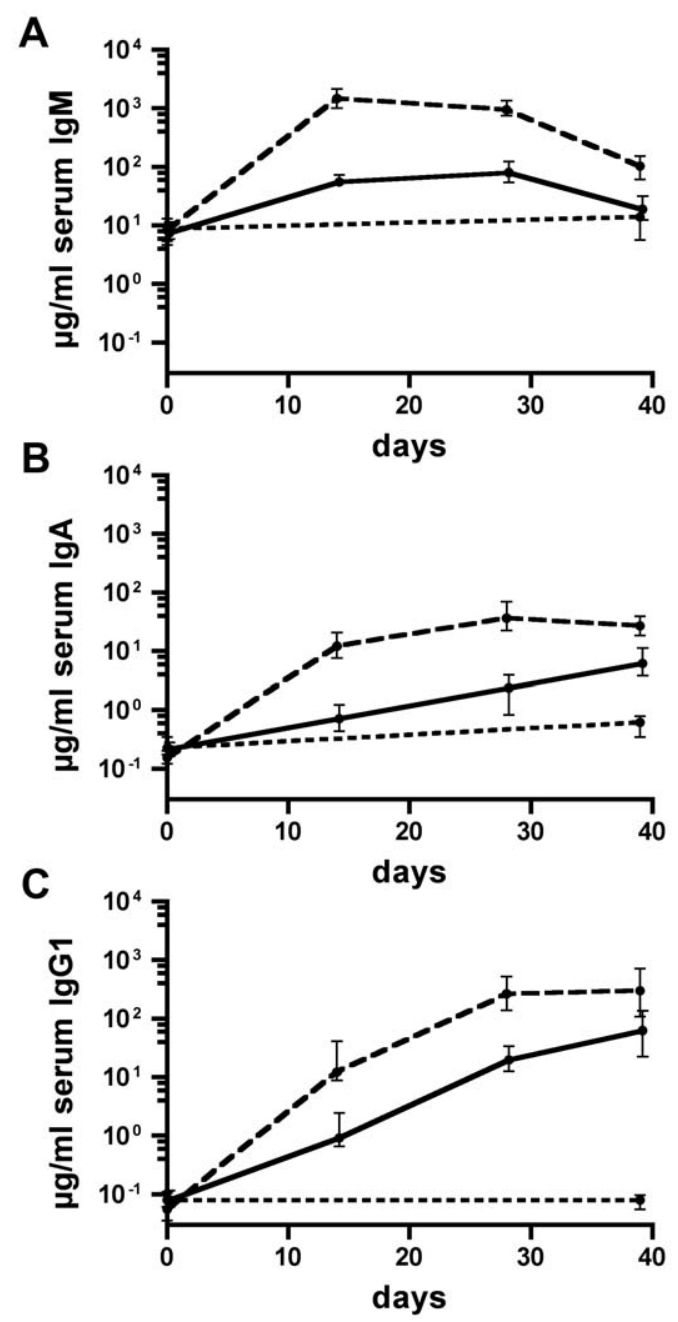

Figure 5. Temporal immune responses to OMVs derived from NTHi strain 3198-R. Shown are the median titers over time of $\lg M(A)$, IgA (B), and IgG1 (C) antibodies to 3198-R OMVs in sera from mice intranasally immunized with either IM-1 (solid line) or IM-2 (dashed line) as well as in sera from nonvaccinated control mice (dotted line) $(n=20$ for each group). The error bars indicate the interquartile range of each data set for each time point.

doi:10.1371/journal.pone.0042664.g005

fold higher infection dose of about $5 \times 10^{5} \mathrm{CFU} /$ mouse. Fig. 10 shows the nasopharyngeal colonization rates in recovered CFU per nasopharynx for all vaccinated and nonvaccinated control mice after challenge with NTHi strain 2019-R (Fig. 10A) or 3198$\mathrm{R}$ (Fig. 10B) for $24 \mathrm{~h}$. All nonvaccinated control mice were stably colonized with median colonization rates of $4 \times 10^{4}$ or $5 \times 10^{4} \mathrm{CFU} /$ nasopharynx for NTHi strains $2019-\mathrm{R}$ or $3198-\mathrm{R}$, respectively. In contrast, both immunization groups challenged with either NTHi strain 2019-R or 3198-R showed significant reductions in their nasopharyngeal colonization rates compared to the nonvaccinated control group $(P<0.05$; Kruskal-Wallis test and post hoc Dunn's multiple comparisons). For both challenge experiments, no significant differences in the colonization rates between the IM-1 and the IM-2 group were observed. The intranasal immunization with IM-1 resulted in 3,900-fold (challenge with 2019-R) and 400-fold (challenge with 3198-R) reduced median colonization rates, whereas the intranasal immunization with IM-2 led to 500-fold and 1,200-fold reductions in the median colonization rates of NTHi strain 2019-R and 3198-R, respec- 
A

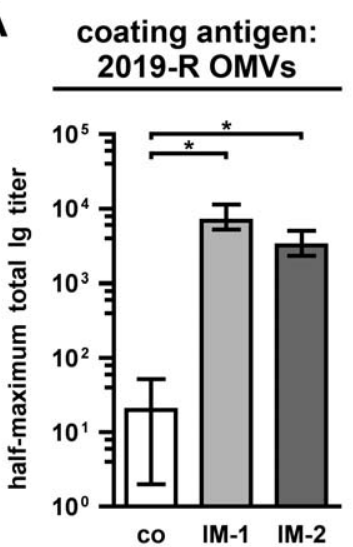

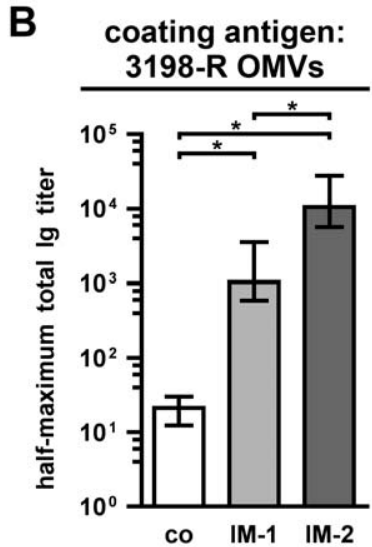

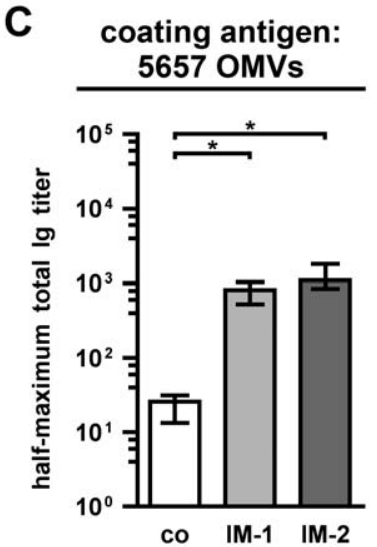

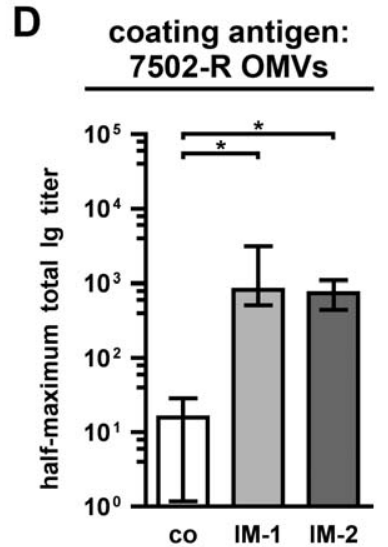

Figure 6. Half-maximum total Ig titers to OMVs derived from homologous and heterologous NTHi strains. Depicted are the median half-maximum total Ig titers to OMVs derived from NTHi strains 2019-R (A), 3198-R (B), 5657 (C), and 7502-R (D) in sera collected at day 39 from mice intranasally immunized with either IM-1 or IM-2 as well as from nonvaccinated control mice (co) $(\mathrm{n}=20$ for each group). The error bars indicate the interquartile range of each data set. Significant differences between the data sets are marked by asterisks $(P<0.05 ;$ Kruskal-Wallis test and post hoc Dunn's multiple comparisons).

doi:10.1371/journal.pone.0042664.g006

tively. Several immunized mice showed no detectable colonization at all. In these cases, the colonization rates were set to the limit of detection of $10 \mathrm{CFU} /$ nasopharynx. In summary, the induced immune responses in mice immunized with either IM-1 or IM-2 are not only protective against a homologous NTHi strain, but also against a heterologous NTHi strain, whose OMVs were not present in the immunization mixtures.

The protective immunity correlates with intranasal immunization and the use of NTHi OMVs

In addition, we performed two complementary immunization experiments to characterize the protective immune response based on NTHi OMVs as vaccine candidates in more detail. Previous immunizations studies using the mouse model and human epidemiological data on otitis media caused by NTHi suggest
A

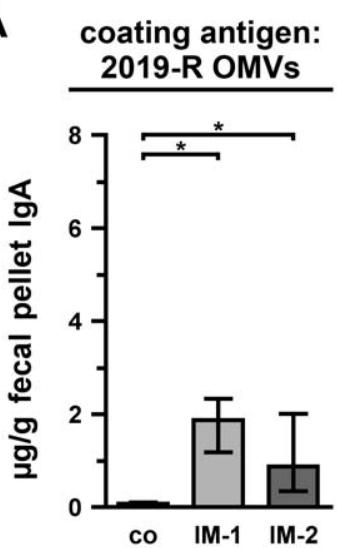

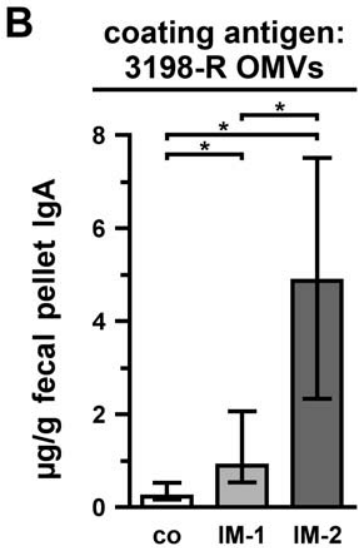

co IM-1 IM-2
Figure 7. Mucosal immune responses to OMVs derived from NTHi strain 2019-R and 3198-R. Shown are the median IgA titers to OMVs derived from NTHi strain 2019-R (A) and 3198-R (B) extracted from fecal pellets collected at day 39 from mice intranasally immunized with either IM-1 or IM-2 as well as from nonvaccinated control mice (co) ( $n=10$ for each group). The error bars indicate the interquartile range of each data set. Significant differences between the data sets are marked by asterisks $(P<0.05$; Kruskal-Wallis test and post hoc Dunn's multiple comparisons).

doi:10.1371/journal.pone.0042664.g007 that IgA, especially secretory IgA in the mucosal surfaces, might be the most important isotype for enhanced clearance of NTHi infections [19,75,76]. Mucosal immunizations like intransasal administration result in a robust $\mathrm{IgA}$ response, whereas immunization via the intraperitoneal route does not induce high IgA levels $[34,57,77,78,79]$. We took advantage of this difference and intraperitoneally immunized mice using OMVs derived from NTHi strain 2019-R (i.p. IM-1). Concordant with the current literature, the intraperitoneally immunized mice induced a robust IgG1 response to OMVs derived from NTHi strain 2019-R, but showed only a small, insignificant induction in IgA levels (Fig. 11A). We also determined the half-maximum total $\mathrm{Ig}$ titers in sera collected at day 39 (Fig. 11B). As expected, the half-maximum total Ig titers in sera of the intraperitoneally immunized mice were significantly higher than those of the nonvaccinated control mice $(P<0.05$; Mann-Whitney $\mathrm{U}$ test $)$. Similar levels in the halfmaximum total Ig titers detected in mice immunized with IM-1 via the intransal or intraperitoenal route indicate that both immunization strategies induced a robust immune response with comparable total antibody levels (Fig. 6A and 11B). In addition, the specificity of the IgG response was analyzed by immunoblot analysis (Fig. 12). Multiple bands in the OMV, OM, and WCL protein profiles derived from the NTHi strain 2019-R were detected using sera collected at day 39 from a representative mouse intraperitoneally immunized with IM-1. Thus, the immune response induced upon intraperitoneal immunization is most likely as diverse as upon intranasal immunization and many components of the OMVs serve as antigens. We challenged the intraperitoneally IM-1 immunized mice with NTHi strain 2019-R and found a significant 25 -fold reduction in the median colonization rate compared to the nonvaccinated control group (Fig. 13, $P<0.05$; Kruskal-Wallis test and post hoc Dunn's multiple comparisons). In contrast to the group receiving IM-1 intranasally, all intraperitoneally immunized mice were colonized with detectable levels and 3-log reduction rates upon challenge with NTHi strain 2019-R could not be reached (Fig. 10 and 13). Thus, the intranasal route of immunization with NTHi OMVs confers higher protection than the intraperitoenal route.

It is very likely that the intranasal immunization with OMVs results in local inflammation and recruitment of phagocytes to the nasopharynx. Thus, such general, unspecific responses of the 
A

IM-1 immunized
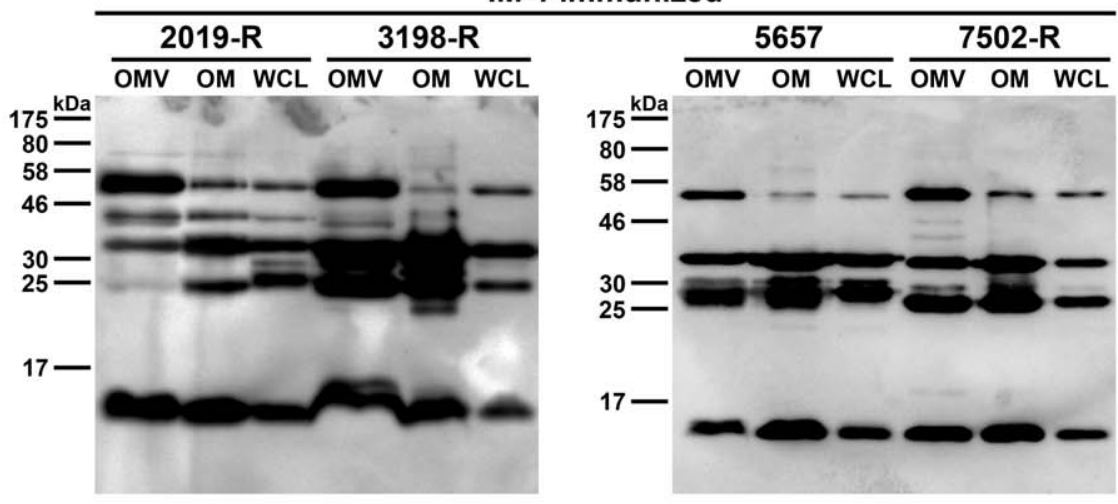

B

IM-2 immunized

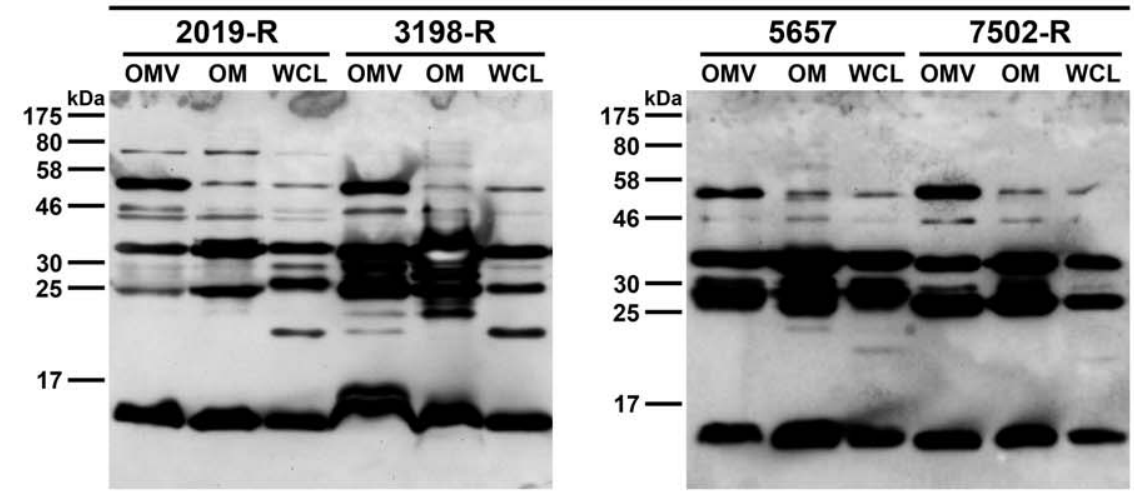

C

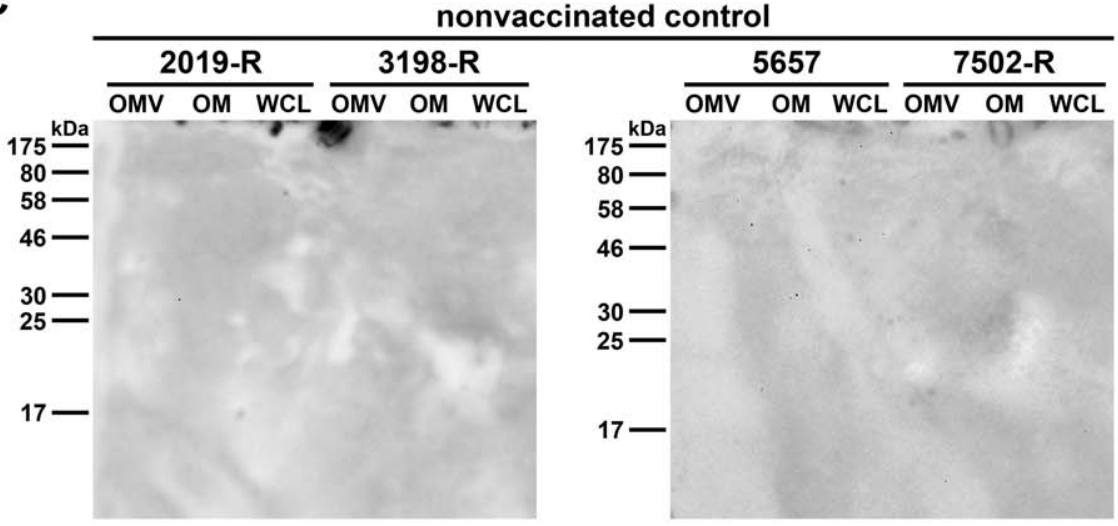

Figure 8. Immunoblot analysis of IgG reactivity in sera from intranasally immunized mice. Representative immunoblots incubated with sera collected at day 39 from mice intranasally immunized with either IM-1 (A) or IM-2 (B) as well as from a nonvaccinated control mouse (C) are shown. Each immunoblot was loaded with OMV, OM, and WCL preparations (approx. $3.2 \mu \mathrm{g}$ protein each) derived from either NTHi strain 2019-R and 3198-R or 5657 and 7502-R. Lines to the left indicate the molecular masses of the protein standards in $\mathrm{kDa}$.

doi:10.1371/journal.pone.0042664.g008

innate immune system could also account for the observed protection. In order to investigate the species specificity of the immune response we performed intranasal immunization studies according to the immunization schedule with OMVs derived from V. cholerae (i.n. Vch-OMV), which have been shown recently to be highly immunogenic and induce a protective immune response against this gastrointestinal pathogen [34,35]. Consistent with these previous studies, the mice induced a robust immune response against $V$. cholerae, which is indicated by a 7000 -fold higher median half-maximum total $\mathrm{Ig}$ titer detected in sera of the V. cholerae OMV immunized mice compared to the nonvaccinated control group (Fig. 14). In contrast, no significant immune response was induced against NTHi strain 2019-R, which was monitored by determination of the half-maximum total $\mathrm{Ig}$ titers and immunoblot analysis using NTHi strain 2019-R OMVs as antigen (data not shown). Challenge of the $V$. cholerae OMV immunized mice with NTHi strain 2019-R revealed only a minor, insignificant 2-fold reduced median colonization rate compared to the nonvaccinated control group (Fig. 13). Thus, the 2- to 3-log reduction of the colonization rates after intranasal immunization with NTHi OMVs could not be reached. In summary, the additional immunization experiments indicate that observed protection against nasopharyngeal colonization with NTHi upon immuniza- 


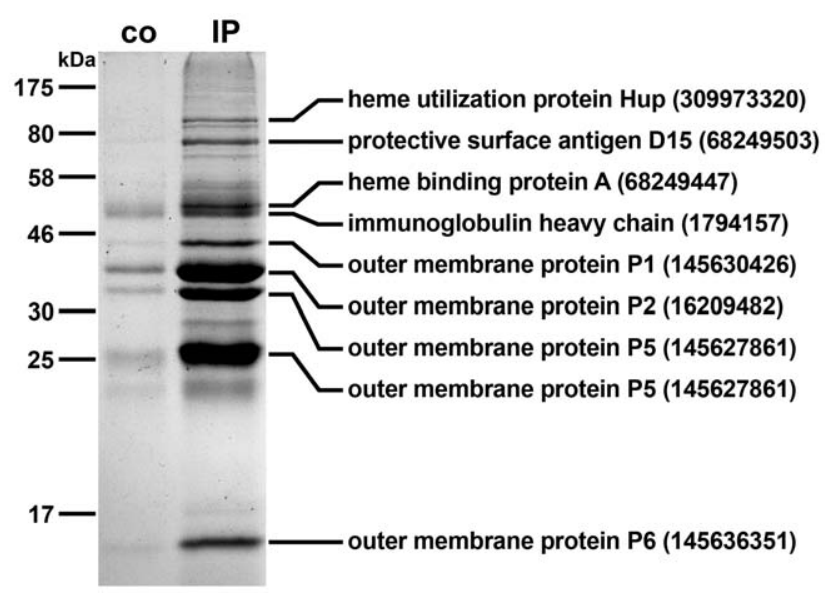

Figure 9. Immunoprecipitation using pooled sera from intranasally IM-1 immunized mice. Kang stained gel showing OMPs that co-immunoprecipitate with serum antibodies from nonvaccinated control mice (co) or antibodies from mice immunized with IM-1 (IP) immobilized onto Dynabeads coupled with protein G. Proteins identified from the IP sample by mass spectrometry are indicated with their respective position on the gel, protein identities and accession numbers on the right.

doi:10.1371/journal.pone.0042664.g009

tion with OMVs highly correlates with the intranasal administration route as well as the use of species-specific OMVs.

\section{Discussion}

The major aim of the present study was to investigate the potential of OMVs derived from NTHi strains to serve as a future vaccine against NTHi infections. OMVs derived from the NTHi strains used in this study were characterized by electron microscopy as well as by OMV and OM profile comparisons. Additionally, we identified eight abundant proteins packaged in the vesicles. Consistent with our analysis, these proteins have also been found in the recently published proteome of OMVs derived from the NTHi strain 86-028NP with the OMPs P2 and P5 being the most abundant proteins [44]. Therefore, this study confirms the production of OMVs by heterologous NTHi strains and provides a protein profile analysis of OMVs released from multiple NTHi strains.

To test the immunogenic and protective properties of NTHi OMVs, we intranasally immunized mice either with IM-1, composed of OMVs derived solely from NTHi strain 2019-R, or with IM-2, an OMV mixture consisting of OMVs derived from NTHi strains 2019-R, 9274-R, and 1479-R. The OMV mixture provided in IM-2 was used to increase the antigen complexity of the NTHi vaccine candidate. We primarily focused on the intranasal route, since nasal immunization is the most effective route to induce a protective immunity in both systemic and mucosal sites [80,81]. This is especially true for the upper respiratory tract [81], which is the primary site of colonization and infection by NTHi [1,3].

Humoral and mucosal immune responses of both immunization groups were monitored by ELISA. In these assays NTHi strain 2019-R was used to determine the immune responses against a homologous strain, whose surface antigens $(\mathrm{OMVs})$ were presented to the immune systems of mice intranasally immunized with IM-1 and IM-2. In contrast, OMVs derived from the heterologous NTHi strain 3198-R were not present in IM-1 or IM-2. It has to be noted that strain $3198-\mathrm{R}$ was allocated into the same LOS group as 9274-R, which was used as a donor for OMVs in IM-2. Nevertheless both strains exhibit distinct differences in the $\mathrm{OM}$ and OMV protein profiles. Thus, the immune systems of mice immunized with IM-2 could have had contact to a similar LOS structure, but not to the same composition of surface protein antigens. In the case of the mice immunized with IM-1, NTHi strain 3198-R served as an unknown strain, whose surface-exposed protein and LOS antigens were never seen by the immune systems. In summary, this experimental design allowed us to determine and analyze the immune response against a homologous strain, against a strain with similar LOS structure, but heterologous protein profile, and against a strain with heterologous protein profile and diverse LOS structure (see Table 1 for details).
A

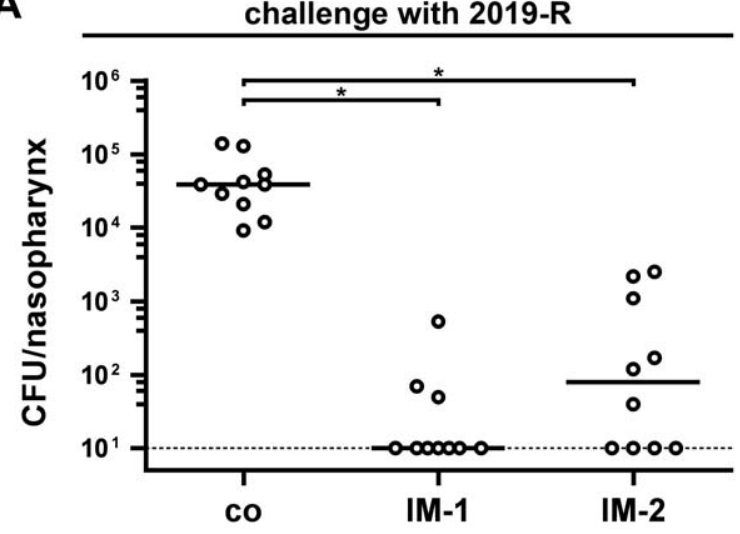

B

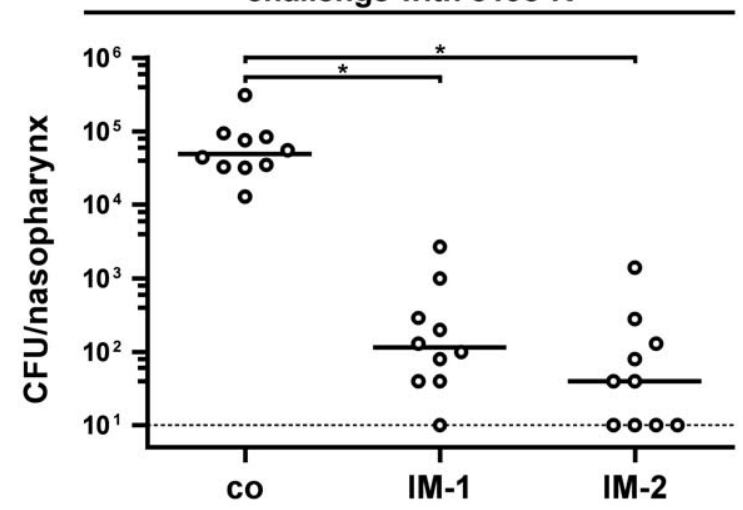

Figure 10. Induced immune responses in mice intranasally immunized with NTHi OMVs are protective against nasopharyngeal challenge. Shown are the nasopharyngeal colonization rates in recovered CFU per nasopharynx for mice intranasally immunized with either IM-1 or IM-2 as well as for nonvaccinated control mice (co). Mice were intranasally challenged with either NTHi strain 2019-R (A) or 3198-R (B) for 24 h. Each circle represents the recovered CFU per nasopharynx from one mouse. The horizontal bars indicate the median of each data set. If no bacteria could be recovered, then the values were set to the limit of detection of $10 \mathrm{CFU} /$ nasopharynx (indicated by the dotted line). Since independent immunization rounds were performed, the exact infection doses ranged from $3.3 \times 10^{5}$ to $6.0 \times 10^{5} \mathrm{CFU} / \mathrm{mouse}$ for NTHi strain $2019-\mathrm{R}$ and from $4.1 \times 10^{5}$ to $4.3 \times 10^{5}$ CFU/mouse for NTHi strain 3198-R. Significant differences between the data sets are marked by asterisks $(P<0.05 ;$ Kruskal-Wallis test and post hoc Dunn's multiple comparisons).

doi:10.1371/journal.pone.0042664.g010 
A

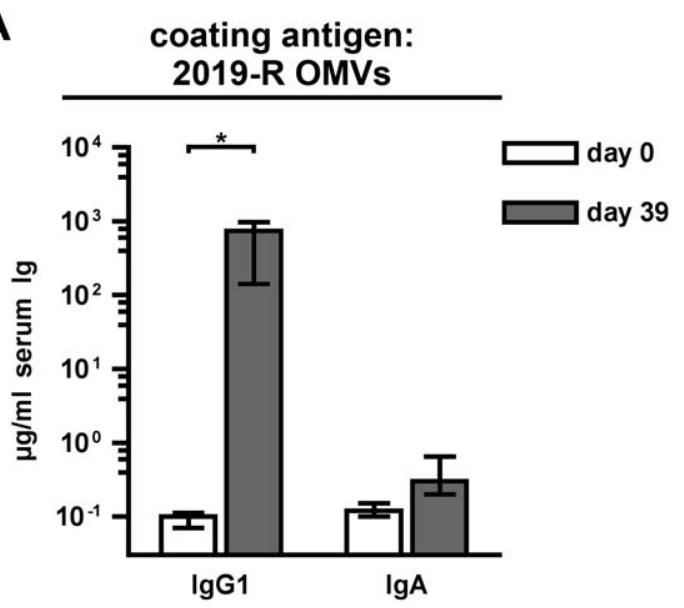

B coating antigen:
2019-R OMVs

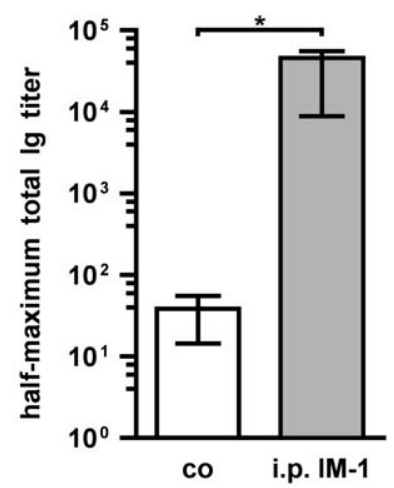

Figure 11. Characterization of the immune response from mice intraperitoneally immunized with NTHi OMVs. (A) Shown are the median IgG1and IgA titers to OMVs derived from NTHi strain 2019-R in sera from mice intraperitoneally immunized with IM-1 collected at day 0 and $39(n=7)$. (B) Shown are the median half-maximum total Ig titers to OMVs derived from NTHi strains 2019-R in sera collected at day 39 from mice intraperitoneally immunized with IM-1 (i.p. IM-1) as well as from nonvaccinated control mice (co) ( $n=6$ for the co group and $n=7$ for the i.p. IM-1 group). The error bars indicate the interquartile range of each data set for each time point. Significant differences between the data sets are marked by asterisks $(P<0.05$; Mann-Whitney $\mathrm{U}$ test).

doi:10.1371/journal.pone.0042664.g011

The levels of the induced immune responses were similar to other successful OMV vaccination studies using OMVs derived from $V$. cholerae or $\mathcal{N}$. meningitidis serogroup B [34,35,57]. The humoral IgA and IgGl responses steadily increased during the whole immunization period, suggesting that the maximum antibody titers were not reached at day 39. Interestingly, the high initial IgM titers have not been detected in comparable studies using OMVs derived from $V$. cholerae $[34,35]$. But the BALB/c mice, which were used in the present study, were not raised under germfree conditions and thus could have been in contact with or colonized with closely related bacterial species like other Pasteurellaceae family members. Since NTHi belongs to the Pasteurellaceae family, it could be suggested that, due to the family relationship, a certain intrinsic level of low-affinity, cross-reacting, natural IgM antibodies to NTHi already exists in BALB/c mice. This

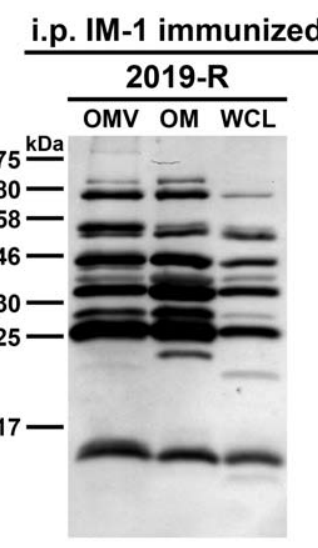

Figure 12. Immunoblot analysis of IgG reactivity in sera from intraperitoneally immunized mice. Shown is a representative immunoblot incubated with sera collected at day 39 from a mouse intraperitoneally immunized with IM-1 (i.p. IM-1). The immunoblot was loaded with OMV, OM, and WCL preparations (approx. $3.2 \mu \mathrm{g}$ protein each) derived from NTHi strain 2019-R. Lines to the left indicate the molecular masses of the protein standards in $\mathrm{kDa}$. doi:10.1371/journal.pone.0042664.g012 assumption is supported by the fact that naive animals produce natural antibodies that mainly belong to the IgM subclass [82,83].

Besides the humoral immune responses, we also determined the induced mucosal immune responses by measuring the secretory IgA titers in fecal pellet extracts. The observed induction of a mucosal immune response against homologous and heterologous NTHi strains is quite an important feature of a potential NTHi vaccine, since the nasopharyngeal mucosa is the first line of defense against respiratory pathogens, such as NTHi [80,81]. In

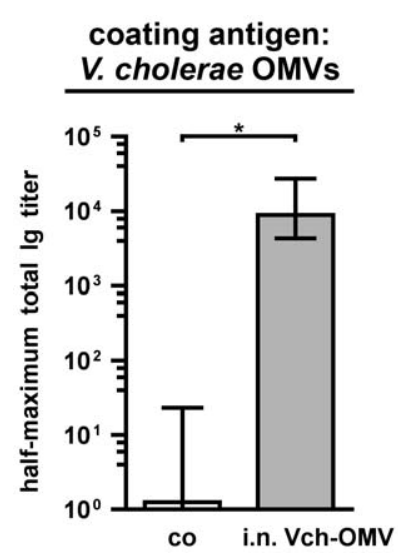

Figure 13. he protective immune responses correlates with intranasal immunization using species-specific OMVs. Shown are the nasopharyngeal colonization rates in recovered CFU per nasopharynx for mice intraperitoneally immunized with NTHi 2019-R (i.p. IM-1) or intranasally with OMVs derived from V. cholerae (i.n. Vch$\mathrm{OMV}$ ) as well as for nonvaccinated control mice (co). Mice were intranasally challenged with NTHi strain 2019-R for 24 h. Each circle represents the recovered CFU per nasopharynx from one mouse. The horizontal bars indicate the median of each data set. The limit of detection for this experiment was $10 \mathrm{CFU} /$ nasopharynx (indicated by the dotted line). The exact infection doses in the independent experiments were approximately $2 \times 10^{5} \mathrm{CFU} /$ mouse. Significant differences between the data sets are marked by asterisks $(P<0.05$; KruskalWallis test and post hoc Dunn's multiple comparisons). doi:10.1371/journal.pone.0042664.g013 


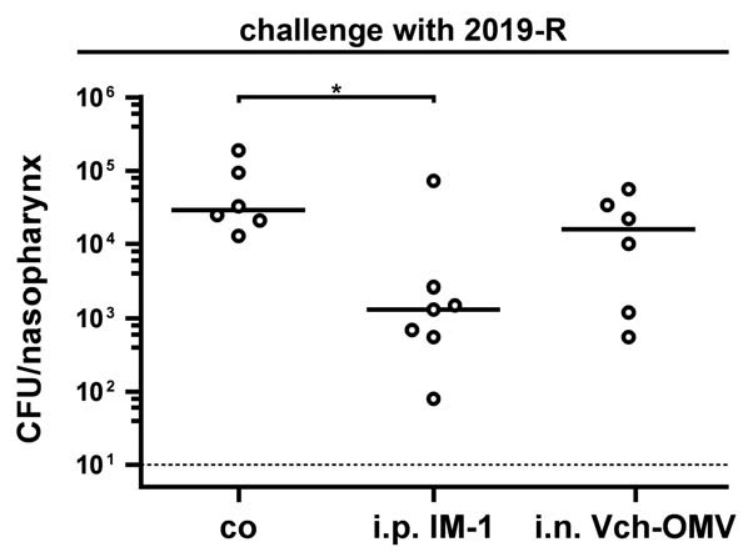

Figure 14. Half maximum total Ig titers in sera from mice intranasally immunized with $V$. cholerae OMVs. Depicted are the median half-maximum total Ig titers to OMVs derived from $V$. cholerae in sera collected at day 39 from mice intranasally immunized with $V$. cholerae OMVs as well as from nonvaccinated control mice (co) $(n=6$ for each group). The error bars indicate the interquartile range of each data set. Significant differences between the data sets are marked by asterisks $(P<0.05$; Mann-Whitney $\mathrm{U}$ test).

doi:10.1371/journal.pone.0042664.g014

general, secretory antibodies to NTHi are detected in body fluids that are obtained either by performing nasal washes using PBS or by collecting saliva after injection of pilocarpine to induce salivary secretion $[19,84]$. However, all mice in this study were intranasally challenged with $\mathrm{NTHi}$, so that nasal washes or pilocarpine injections most likely would have interfered with nasopharyngeal colonization. Therefore, we used fecal pellets to determine the mucosal immune responses, because it has been shown before that secretory IgA antibodies reflecting the mucosal immune response can also be found in feces and that IgA titers in feces correlate with those in saliva $[35,85,86]$.

By comparing the elicited humoral and mucosal immune responses as well as the half-maximum total Ig titers against the homologous NTHi strain 2019-R, it became obvious that the use of an OMV mixture in IM-2 is no disadvantage for the induction of a high-titer immune response, although just a third of the amount of 2019-R OMVs were present in this immunization mixture compared to IM-1. Furthermore, mice immunized with IM-2 exhibited higher immune responses against the heterologous NTHi strain 3198-R compared to the IM-1 immunized group throughout the entire study. IM-2 contained OMVs derived from NTHi strain 9274-R, which belongs to the same LOS group as NTHI strain 3198-R. Thus, mice immunized with IM-2 might have raised antibodies against type III LOS structures. This would consequently result in a cross-reaction with LOS of NTHI 3198-R and may explain the observed immune response patterns against NTHi strain 3198-R. Therefore, we also determined the halfmaximum total Ig titers against the NTHi strains 5657 and 7502$\mathrm{R}$, which belong to the LOS groups IV and $\mathrm{V}$, respectively. Although neither IM-1 nor IM-2 contained OMVs derived from a type IV or V NTHi strain, mice of both immunization groups showed a significant immune response against these strains. This indicates that intranasal immunization with $\mathrm{OMV}$ s derived from just one NTHi strain is sufficient to induce a robust humoral immune response against heterologous and unknown NTHi strains.

Immunoblot analyses were performed to characterize the complexity of the induced immune response. Multiple bands in the OMV, OM, and WCL protein profiles were detected not only in preparations derived from the homologous NTHi strain 2019$\mathrm{R}$, but also in preparations derived from the heterologous NTHi strains 3198-R, 5657, and 7502-R. Similar results have also been reported by immunization studies using OMVs derived from $V$. cholerae and Acinetobacter baumannii [34,87]. Interestingly, already mice immunized with IM-1, just containing one OMV type, induced a quite diverse immune response against variety of antigens including even those from heterologous NTHi strains unknown to the immune system. Immunoprecipitation revealed several important immunogenic proteins. Heme utilization protein and heme binding protein A are both involved in heme utilization, which is a growth requirement for $H$. influenzae and affects virulence $[88,89,90]$. Furthermore, protective surface antigen D15 as well as the OMPs P1, P2, P5 and P6 have already been independently suggested as promising antigens for NTHi vaccine candidates $[13,14,16,91,92,93]$. Thus, NTHi OMVs contain numerous immunogenic proteins and allow the combined presentation of these antigens to the host strengthening their potential as a candidate for NTHi vaccine development.

The protective capacity of the induced immune responses is highlighted by the intranasal challenge of the mice with either the homologous NTHi strain 2019-R or the heterologous NTHi strain 3198-R. Regardless of the NTHi strain challenged with, groups intranasally immunized with NTHi OMVs showed significant reductions in their nasopharyngeal colonization rates compared to the nonvaccinated control group, ranging from 400 to 3,900-fold. To our knowledge, this is the first mouse study reporting such high reduction rates for nasopharyngeal colonization studies with NTHi after immunization with a potential NTHi vaccine. We analyzed the protective immune response in more detail and performed two control immunization experiments. We changed the administration route and intraperitoneally immunized mice with IM-1 to avoid induction of a IgA response. These intraperitoneally immunized group exhibited a significant reduction of the nasopharyngeal colonization, but the median colonization rate in intraperitoneally immunized mice was still 100-fold higher compared to the group intranasally immunized with NTHi OMVs. Thus, the best protection upon immunization with NTHi OMVs was observed via the mucosal administration route. Although we cannot exclude other mechanisms, it would be concordant with epidemiological studies on NTHi infections that the IgA response, especially the levels of secretory IgA, are the most important antibodies for the protection against NTHi infections [75,76]. Besides the induction of a robust antibody response, intranasal immunizations with OMVs will most likely also stimulate antibody-independent mechanism of the immune system, i.e. inflammation, cytokine secretion and recruitment of phagocytes. To investigate the potential role of such general, unspecific responses of the innate immune system we performed intranasal immunizations using OMVs from $V$. cholerae, which is only distantly related to NTHi. Consequently, these mice induced a robust antibody response against $V$. cholerae, but not against NTHi. Since $V$. cholerae OMV immunized mice failed to show protection against challenge with NTHi, nonspecific mechanisms of the immune system cannot account for the reduced colonization observed upon immunization with NTHi OMVs. Consequently, the protective immune response mainly depends on the use of NTHi OMVs and is therefore specific to the OMVs present in the vaccine candidate. Recently, an antibody-independent, $\mathrm{CD} 4^{+}$ dependent protective immunity against pneumococcal colonization was described after mucosal immunization using a pneumococcal whole cell killed vaccine [94,95]. At the current stage similar effects, which are antibody-independent, but specific to NTHi OMVs, cannot be excluded for our model and future 
investigations using for example antibody-deficient mice will be necessary to investigate all facets of the protective immunity in more detail.

As mentioned above, IM-2 contained OMVs from NTHI strains allocated into the LOS groups I, II and III. Consequently, these mice could have raised immunoglobulins directed against the LOS structures of the NTHI strains 2019-R (type II) and 3198-R (type III) used for the challenge. Thus, we cannot exclude that the observed protection for the IM-2 immunization group relies on anti-LOS antibodies. However, this does not explain the robust induced immune response against heterologous NTHi strains and protection against NTHI strain 3198-R of mice immunized with IM-1, containing solely OMVs derived from NTHI strain 2019-R. Additionally, a recent study by Hirano et al. investigated the protective immune response upon immunization with a LOSbased conjugate vaccine candidate in the mouse model [19]. Immunized mice showed only a 2 - to 4-fold reduction in nasopharyngeal colonization after challenge with homologous or heterologous NTHi strains compared to the control group. The higher reduction rates observed in the present study suggest that the protective immune response of the OMV-based vaccine candidate does not depend on antibodies against the LOS structures. Hence, immunization with OMVs results in crossprotection between different LOS groups and the LOS is most likely not the dominant protective antigen.

This is in contrast to a recent OMV immunization study, demonstrating that LPS is the major protective antigen of a $V$. cholerae vaccine candidate based on OMVs and that no crossserogroup protection can be achieved by just using OMVs derived from one $V$. cholerae serogroup in the immunization mixture [32]. One explanation might be that the LOS of NTHi strains is much shorter compared to the $V$. cholerae LPS with relatively long and variable $\mathrm{O}$-antigens, which might act as spacers between the surface antigens associated with the $\mathrm{OM}$ and the respective antibodies.

Like any other animal model, the NTHi mouse model cannot reflect all parts of the human infection. A limitation is the lack of a chronic pulmonary colonization due to rapid clearing of the bacteria in the respiratory tract. Nevertheless, the mouse model is well established in the field and has been used in the past to study several NTHi vaccine candidates $[15,19,96]$. This study should be seen as a first report characterizing a new vaccine candidate based

\section{References}

1. Erwin AL, Smith AL (2007) Nontypeable Haemophilus influenzae: understanding virulence and commensal behavior. Trends Microbiol 15: 355-362.

2. Johnston JW, Apicella MA (2009) Haemophilus influenzae. In: Schaechter M, editor. Encyclopedia of Microbiology, 3rd ed. Amsterdam: Academic Press, Elsevier Ltd. pp. 153-162.

3. Rao VK, Krasan GP, Hendrixson DR, Dawid S, St Geme JW 3rd (1999) Molecular determinants of the pathogenesis of disease due to non-typable Haemophilus influenzae. FEMS Microbiol Rev 23: 99-129.

4. Murphy TF (2006) The role of bacteria in airway inflammation in exacerbations of chronic obstructive pulmonary disease. Curr Opin Infect Dis 19: 225-230.

5. Sethi S, Murphy TF (2001) Bacterial infection in chronic obstructive pulmonary disease in 2000: a state-of-the-art review. Clin Microbiol Rev 14: 336-363.

6. WHO (2008) World health statistics 2008. Geneva, Switzerland: World Health Organization. Available: http://www.who.int/gho/publications/world_health_ statistics/EN_WHS08_Full.pdf. Accessed 2012 Jul 14.

7. Morris SK, Moss WJ, Halsey N (2008) Haemophilus influenzae type b conjugate vaccine use and effectiveness. Lancet Infect Dis 8: 435-443.

8. Adam HJ, Richardson SE, Jamieson FB, Rawte P, Low DE, et al. (2010) Changing epidemiology of invasive Haemophilus influenzae in Ontario, Canada: evidence for herd effects and strain replacement due to Hib vaccination. Vaccine 28: 4073-4078.

9. Dworkin MS, Park L, Borchardt SM (2007) The changing epidemiology of invasive Haemophilus influenzae disease, especially in persons $>$ or $=65$ years old. Clin Infect Dis 44: 810-816. on OMVs, which induces cross-protection against heterologous NTHi strains. Future work has to investigate, if the initial results of the present study using the mouse model also hold true in the human system. Noteworthy, in the case of otitis media caused by NTHi, several studies suggest a correlation between the presence of serum antibodies against NTHi and protection [75,76,97,98].

In summary, this study has confirmed that NTHi strains release OMVs and has shown that these OMVs have a high potential to act as vaccine against NTHi infections. We have demonstrated that already an intranasal immunization with OMVs derived from one NTHi strain results in a robust and complex humoral, mucosal, and protective immune response against homologous and heterologous NTHi strains, even without the use of a mucosal adjuvant. Obviously, OMVs naturally contain a balanced mixture of immunogenic, protective antigens and adjuvants. OMVs can be isolated from different donor strains and easily combined in OMV mixtures. Based on the data of this study, the use of an OMV mixture is not necessary, but also has no disadvantage for the induction of a protective immune response against NTHi strains. It can be speculated that the use of a heterogeneous OMV mixture might be advantageous by inducing a more complex immune response against different antigens of NTHi strains. Additionally, one could extend this idea and create a combined vaccine candidate against several Gram-negative pathogens by combining OMVs derived from different donor species. Thus, the increased antigenic diversity of an OMV mixture could further stimulate the induction of cross-reacting antibodies, which in turn may help to develop a broad-spectrum vaccine not only against heterologous NTHi strains, but also against other Gram-negative pathogens of interest.

\section{Acknowledgments}

We thank Michael A. Apicella for providing NTHi strains 1479, 2019, $3198,5657,7502$, and 9274 as well as Bela Reiz for assistance with the mass spectrometry.

\section{Author Contributions}

Conceived and designed the experiments: SR DL KS JR SS. Performed the experiments: SR DL JI KS SS. Analyzed the data: SR DL JI KS MFF JR SS. Contributed reagents/materials/analysis tools: MFF GK JR SS. Wrote the paper: SR SS.

10. Tsang RS, Sill ML, Skinner SJ, Law DK, Zhou J, et al. (2007) Characterization of invasive Haemophilus influenzae disease in Manitoba, Canada, 2000-2006: invasive disease due to non-type b strains. Clin Infect Dis 44: 1611-1614.

11. Ulanova M, Tsang RS (2009) Invasive Haemophilus influenzae disease: changing epidemiology and host-parasite interactions in the 21 st century. Infect Genet Evol 9: 594-605.

12. van Alphen L, Caugant DA, Duim B, O'Rourke M, Bowler LD (1997) Differences in genetic diversity of nonecapsulated Haemophilus influenzae from various diseases. Microbiology 143: 1423-1431.

13. Poolman JT, Bakaletz L, Cripps A, Denoel PA, Forsgren A, et al. (2000) Developing a nontypeable Haemophilus influenzae (NTHi) vaccine. Vaccine 19 Suppl 1: S108-S115.

14. Ostberg KL, Russell MW, Murphy TF (2009) Mucosal immunization of mice with recombinant OMP P2 induces antibodies that bind to surface epitopes of multiple strains of nontypeable Haemophilus influenzae. Mucosal Immunol 2: 6373.

15. Hotomi M, Ikeda Y, Suzumoto M, Yamauchi K, Green BA, et al. (2005) A recombinant $\mathrm{P} 4$ protein of Haemophilus influenzae induces specific immune responses biologically active against nasopharyngeal colonization in mice after intranasal immunization. Vaccine 23: 1294-1300.

16. Murphy TF, Kirkham C, Lesse AJ (2006) Construction of a mutant and characterization of the role of the vaccine antigen P6 in outer membrane integrity of nontypeable Haemophilus influenzae. Infect Immun 74: 5169-5176.

17. Murphy TF (2009) Current and future prospects for a vaccine for nontypeable Haemophilus influenzae. Curr Infect Dis Rep 11: 177-182. 
18. Singh B, Brant M, Kilian M, Hallstrom B, Riesbeck K (2010) Protein E of Haemophilus influenzae is a ubiquitous highly conserved adhesin. J Infect Dis 201: 414-419.

19. Hirano T, Hou Y, Jiao X, Gu XX (2003) Intranasal immunization with a lipooligosaccharide-based conjugate vaccine from nontypeable Haemophilus influenzae enhances bacterial clearance in mouse nasopharynx. FEMS Immunol Med Microbiol 35: 1-10.

20. Wu T, Chen J, Murphy TF, Green BA, Gu XX (2005) Investigation of nontypeable Haemophilus influenzae outer membrane protein P6 as a new carrier for lipooligosaccharide conjugate vaccines. Vaccine 23: 5177-5185.

21. Gu XX, Tsai CM, Ueyama T, Barenkamp SJ, Robbins JB, et al. (1996) Synthesis, characterization, and immunologic properties of detoxified lipooligosaccharide from nontypeable Haemophilus influenzae conjugated to proteins. Infect Immun 64: 4047-4053.

22. Chang A, Kaur R, Michel LV, Casey JR, Pichichero M (2011) Haemophilus influenzae vaccine candidate outer membrane protein $\mathrm{P} 6$ is not conserved in all strains. Hum Vaccin 7: 102-105.

23. Michel LV, Kalmeta B, McCreary M, Snyder J, Craig P, et al. (2011) Vaccine candidate $\mathrm{P} 6$ of nontypable Haemophilus influenzae is not a transmembrane protein based on protein structural analysis. Vaccine 29: 1624-1627.

24. Beveridge TJ (1999) Structures of gram-negative cell walls and their derived membrane vesicles. J Bacteriol 181: 4725-4733.

25. Ellis TN, Kuehn MJ (2010) Virulence and immunomodulatory roles of bacterial outer membrane vesicles. Microbiol Mol Biol Rev 74: 81-94.

26. Mashburn-Warren LM, Whiteley M (2006) Special delivery: vesicle trafficking in prokaryotes. Mol Microbiol 61: 839-846.

27. Kulp A, Kuehn MJ (2010) Biological functions and biogenesis of secreted bacterial outer membrane vesicles. Annu Rev Microbiol 64: 163-184.

28. Gankema H, Wensink J, Guinee PA, Jansen WH, Witholt B (1980) Some characteristics of the outer membrane material released by growing enterotoxigenic Escherichia coli. Infect Immun 29: 704-713.

29. Hoekstra D, van der Laan JW, de Leij L, Witholt B (1976) Release of outer membrane fragments from normally growing Escherichia coli. Biochim Biophys Acta 455: 889-899.

30. Post DM, Zhang D, Eastvold JS, Teghanemt A, Gibson BW, et al. (2005) Biochemical and functional characterization of membrane blebs purified from Neisseria meningitidis serogroup B. J Biol Chem 280: 38383-38394.

31. Kim SH, Kim KS, Lee SR, Kim E, Kim MS, et al. (2009) Structural modifications of outer membrane vesicles to refine them as vaccine delivery vehicles. Biochim Biophys Acta 1788: 2150-2159.

32. Bishop AL, Schild S, Patimalla B, Klein B, Camilli A (2010) Mucosal immunization with Vibrio cholerae outer membrane vesicles provides maternal protection mediated by antilipopolysaccharide antibodies that inhibit bacterial motility. Infect Immun 78: 4402-4420.

33. Holst J, Martin D, Arnold R, Huergo CC, Oster P, et al. (2009) Properties and clinical performance of vaccines containing outer membrane vesicles from Neisseria meningitidis. Vaccine 27 Suppl 2: B3-B12.

34. Schild S, Nelson EJ, Camilli A (2008) Immunization with Vibrio cholerae outer membrane vesicles induces protective immunity in mice. Infect Immun 76: $4554-4563$.

35. Schild S, Nelson EJ, Bishop AL, Camilli A (2009) Characterization of Vibrio cholerae outer membrane vesicles as a candidate vaccine for cholera. Infect Immun 77: 472-484

36. Alaniz RC, Deatherage BL, Lara JC, Cookson BT (2007) Membrane vesicles are immunogenic facsimiles of Salmonella typhimurium that potently activate dendritic cells, prime $\mathrm{B}$ and $\mathrm{T}$ cell responses, and stimulate protective immunity in vivo. J Immunol 179: 7692-7701.

37. Whitmire WM, Garon CF (1993) Specific and nonspecific responses of murine B cells to membrane blebs of Borrelia burgdorferi. Infect Immun 61: 1460-1467.

38. Roberts R, Moreno G, Bottero D, Gaillard ME, Fingermann M, et al. (2008) Outer membrane vesicles as acellular vaccine against pertussis. Vaccine 26 : 4639-4646.

39. Kesavalu L, Ebersole JL, Machen RL, Holt SC (1992) Porphyromonas gingivalis virulence in mice: induction of immunity to bacterial components. Infect Immun 60: $1455-1464$.

40. Sexton K, Lennon D, Oster P, Crengle S, Martin D, et al. (2004) The New Zealand Meningococcal Vaccine Strategy: a tailor-made vaccine to combat a devastating epidemic. N Z Med J 117: U1015.

41. Deich RA, Hoyer LC (1982) Generation and release of DNA-binding vesicles by Haemophilus influenzae during induction and loss of competence. J Bacteriol 152: 855-864.

42. Concino MF, Goodgal SH (1982) DNA-binding vesicles released from the surface of a competence-deficient mutant of Haemophilus influenzae. J Bacteriol 152: $441-450$.

43. Hong W, Pang B, West-Barnette S, Swords WE (2007) Phosphorylcholine expression by nontypeable Haemophilus influenzae correlates with maturation of biofilm communities in vitro and in vivo. J Bacteriol 189: 8300-8307.

44. Sharpe SW, Kuehn MJ, Mason KM (2011) Elicitation of epithelial cell-derived immune effectors by outer membrane vesicles of nontypeable Haemophilus influenzae. Infect Immun 79: 4361-4369.

45. Schild S, Tamayo R, Nelson EJ, Qadri F, Calderwood SB, et al. (2007) Genes induced late in infection increase fitness of Vibrio cholerae after release into the environment. Cell Host Microbe 2: 264-277.
46. Murphy TF, Apicella MA (1985) Antigenic heterogeneity of outer membrane proteins of nontypable Haemophilus influenzae is a basis for a serotyping system. Infect Immun 50: 15-21.

47. Meats E, Feil EJ, Stringer S, Cody AJ, Goldstein R, et al. (2003) Characterization of encapsulated and noncapsulated Haemophilus influenzae and determination of phylogenetic relationships by multilocus sequence typing. J Clin Microbiol 41: 1623-1636.

48. Carlone GM, Thomas ML, Rumschlag HS, Sottnek FO (1986) Rapid microprocedure for isolating detergent-insoluble outer membrane proteins from Haemophilus species. J Clin Microbiol 24: 330-332.

49. Campagnari AA, Gupta MR, Dudas KC, Murphy TF, Apicella MA (1987) Antigenic diversity of lipooligosaccharides of nontypable Haemophilus influenzae. Infect Immun 55: 882-887.

50. Gaucher SP, Cancilla MT, Phillips NJ, Gibson BW, Leary JA (2000) Mass spectral characterization of lipooligosaccharides from Haemophilus influenzae 2019. Biochemistry 39: 12406-12414.

51. Phillips NJ, Apicella MA, Griffiss JM, Gibson BW (1992) Structural characterization of the cell surface lipooligosaccharides from a nontypable strain of Haemophilus influenzae. Biochemistry 31: 4515-4526.

52. Engskog MK, Deadman M, Li J, Hood DW, Schweda EK (2011) Detailed structural features of lipopolysaccharide glycoforms in nontypeable Haemophilus influenzae strain 2019. Carbohydr Res 346: 1241-1249.

53. Pang B, Winn D, Johnson R, Hong W, West-Barnette S, et al. (2008) Lipooligosaccharides containing phosphorylcholine delay pulmonary clearance of nontypeable Haemophilus influenzae. Infect Immun 76: 2037-2043.

54. Tong HH, Blue LE, James MA, Chen YP, DeMaria TF (2000) Evaluation of phase variation of nontypeable Haemophilus influenzae lipooligosaccharide during nasopharyngeal colonization and development of otitis media in the chinchilla model. Infect Immun 68: 4593-4597.

55. Rahman MM, Gu XX, Tsai CM, Kolli VS, Carlson RW (1999) The structural heterogeneity of the lipooligosaccharide (LOS) expressed by pathogenic nontypeable Haemophilus influenzae strain NTHi 9274. Glycobiology 9: 1371-1380.

56. Dalseg R, Wedege E, Holst J, Haugen IL, Hoiby EA, et al. (1999) Outer membrane vesicles from group $\mathbf{B}$ meningococci are strongly immunogenic when given intranasally to mice. Vaccine 17: 2336-2345.

57. Saunders NB, Shoemaker DR, Brandt BL, Moran EE, Larsen T, et al. (1999) Immunogenicity of intranasally administered meningococcal native outer membrane vesicles in mice. Infect Immun 67: 113-119.

58. Laemmli UK (1970) Cleavage of structural proteins during the assembly of the head of bacteriophage T4. Nature 227: 680-685.

59. Kang D, Gho YS, Suh M, Kang C (2002) Highly sensitive and fast protein detection with coomassie brilliant blue in sodium dodecyl sulfate-polyacrylamide gel electrophoresis. Bull Kor Chem Soc 23: 1511-1512.

60. Shevchenko A, Wilm M, Vorm O, Mann M (1996) Mass spectrometric sequencing of proteins silver-stained polyacrylamide gels. Anal Chem 68: 850 858.

61. Gu XX, Rudy SF, Chu C, McCullagh L, Kim HN, et al. (2003) Phase I study of a lipooligosaccharide-based conjugate vaccine against nontypeable Haemophilus influenzae. Vaccine 21: 2107-2114.

62. Hong W, Peng D, Rivera M, Gu XX (2010) Protection against nontypeable Haemophilus influenzae challenges by mucosal vaccination with a detoxified lipooligosaccharide conjugate in two chinchilla models. Microbes Infect 12: 1118.

63. Gu XX, Sun J, Jin S, Barenkamp SJ, Lim DJ, et al. (1997) Detoxified lipooligosaccharide from nontypeable Haemophilus influenzae conjugated to proteins confers protection against otitis media in chinchillas. Infect Immun 65: $4488-4493$.

64. Bauman SJ, Kuehn MJ (2006) Purification of outer membrane vesicles from Pseudomonas aeruginosa and their activation of an IL-8 response. Microbes Infect 8: 2400-2408.

65. Haurat MF, Aduse-Opoku J, Rangarajan M, Dorobantu L, Gray MR, et al. (2011) Selective sorting of cargo proteins into bacterial membrane vesicles. J Biol Chem 286: 1269-1276.

66. Olofsson A, Vallstrom A, Petzold K, Tegtmeyer N, Schleucher J, et al. (2010) Biochemical and functional characterization of Helicobacter pylori vesicles. Mol Microbiol 77: 1539-1555.

67. Kato S, Kowashi Y, Demuth DR (2002) Outer membrane-like vesicles secreted by Actinobacillus actinomycetemcomitans are enriched in leukotoxin. Microb Pathog 32: $1-13$.

68. Wensink J, Witholt B (1981) Outer-membrane vesicles released by normally growing Escherichia coli contain very little lipoprotein. Eur J Biochem 116: 331335.

69. Barenkamp SJ, Munson RS Jr, Granoff DM (1982) Outer membrane protein and biotype analysis of pathogenic nontypable Haemophilus influenzae. Infect Immun 36: 535-540.

70. Groeneveld K, van Alphen L, Eijk PP, Jansen HM, Zanen HC (1988) Changes in outer membrane proteins of nontypable Haemophilus influenzae in patients with chronic obstructive pulmonary disease. J Infect Dis 158: 360-365.

71. Groeneveld K, van Alphen L, Voorter C, Eijk PP, Jansen HM, et al. (1989) Antigenic drift of Haemophilus influenzae in patients with chronic obstructive pulmonary disease. Infect Immun 57: 3038-3044.

72. Loos BG, Bernstein JM, Dryja DM, Murphy TF, Dickinson DP (1989) Determination of the epidemiology and transmission of nontypable Haemophilus 
influenzae in children with otitis media by comparison of total genomic DNA restriction fingerprints. Infect Immun 57: 2751-2757.

73. Murphy TF, Dudas KC, Mylotte JM, Apicella MA (1983) A subtyping system for nontypable Haemophilus influenzae based on outer-membrane proteins. J Infect Dis 147: 838-846.

74. Spinola SM, Peacock J, Denny FW, Smith DL, Cannon JG (1986) Epidemiology of colonization by nontypable Haemophilus influenzae in children: a longitudinal study. J Infect Dis 154: 100-109.

75. Ogra PL, Bernstein JM, Yurchak AM, Coppola PR, Tomasi TB Jr (1974) Characteristics of secretory immune system in human middle ear: implications in otitis media. J Immunol 112: 488-495.

76. Sloyer JL Jr, Howie VM, Ploussard JH, Schiffman G, Johnston RB Jr (1976) Immune response to acute otitis media: association between middle ear fluid antibody and the clearing of clinical infection. J Clin Microbiol 4: 306-308.

77. McGhee JR, Mestecky J, Dertzbaugh MT, Eldridge JH, Hirasawa M, et al. (1992) The mucosal immune system: from fundamental concepts to vaccine development. Vaccine 10: 75-88.

78. Mestecky J, McGhee JR (1992) Prospects for human mucosal vaccines. Adv Exp Med Biol 327: 13-23.

79. Belyakov IM, Ahlers JD (2009) What role does the route of immunization play in the generation of protective immunity against mucosal pathogens? J Immunol 183: 6883-6892.

80. Holmgren J, Czerkinsky C (2005) Mucosal immunity and vaccines. Nat Med 11: S45-53.

81. Kiyono H, Fukuyama S (2004) NALT- versus Peyer's-patch-mediated mucosal immunity. Nat Rev Immunol 4: 699-710.

82. Avrameas S (1991) Natural autoantibodies: from 'horror autotoxicus' to 'gnothi seauton'. Immunol Today 12: 154-159.

83. Ochsenbein AF, Fehr T, Lutz G, Suter M, Brombacher F, et al. (1999) Control of early viral and bacterial distribution and disease by natural antibodies. Science 286: 2156-2159.

84. Bertot GM, Becker PD, Guzman CA, Grinstein S (2004) Intranasal vaccination with recombinant P6 protein and adamantylamide dipeptide as mucosal adjuvant confers efficient protection against otitis media and lung infection by nontypeable Haemophilus influenzae. J Infect Dis 189: 1304-1312.

85. Hirano T, Jiao X, Chen Z, Van Waes C, Gu XX (2006) Kinetics of mouse antibody and lymphocyte responses during intranasal vaccination with a lipooligosaccharide-based conjugate vaccine. Immunol Lett 107: 131-139.

86. Vetvik H, Grewal HM, Haugen IL, Ahren C, Haneberg B (1998) Mucosal antibodies can be measured in air-dried samples of saliva and feces. J Immunol Methods 215: 163-172.
87. McConnell MJ, Rumbo C, Bou G, Pachon J (2011) Outer membrane vesicles as an acellular vaccine against Acinetobacter baumannii. Vaccine 29: 5705-5710.

88. Morton DJ, Madore LL, Smith A, Vanwagoner TM, Seale TW, et al. (2005) The heme-binding lipoprotein $(\mathrm{HbpA})$ of Haemophilus influenzae: role in heme utilization. FEMS Microbiol Lett 253: 193-199.

89. Morton DJ, Seale TW, Bakaletz LO, Jurcisek JA, Smith A, et al. (2009) The heme-binding protein (HbpA) of Haemophilus influenzae as a virulence determinant. Int J Med Microbiol 299: 479-488.

90. Morton DJ, Smith A, Ren Z, Madore LL, VanWagoner TM, et al. (2004) Identification of a haem-utilization protein (Hup) in Haemophilus influenzae. Microbiology 150: 3923-3933.

91. Loosmore SM, Yang YP, Coleman DC, Shortreed JM, England DM, et al. (1997) Outer membrane protein D15 is conserved among Haemophilus influenzae species and may represent a universal protective antigen against invasive disease. Infect Immun 65: 3701-3707.

92. Bolduc GR, Bouchet V, Jiang RZ, Geisselsoder J, Truong-Bolduc QC, et al. (2000) Variability of outer membrane protein P1 and its evaluation as a vaccine candidate against experimental otitis media due to nontypeable Haemophilus influenzae: an unambiguous, multifaceted approach. Infect Immun 68: 45054517.

93. Novotny LA, Partida-Sanchez S, Munson RS Jr, Bakaletz LO (2008) Differential uptake and processing of a Haemophilus influenzae P5-derived immunogen by chinchilla dendritic cells. Infect Immun 76: 967-977.

94. Malley R, Trzcinski K, Srivastava A, Thompson CM, Anderson PW, et al. (2005) CD4+ T cells mediate antibody-independent acquired immunity to pneumococcal colonization. Proc Natl Acad Sci U S A 102: 4848-4853.

95. Malley R, Anderson PW (2012) Serotype-independent pneumococcal experimental vaccines that induce cellular as well as humoral immunity. Proc Natl Acad Sci U S A 109: 3623-3627.

96. Mason KW, Zhu D, Scheuer CA, McMichael JC, Zlotnick GW, et al. (2004) Reduction of nasal colonization of nontypeable Haemophilus influenzae following intranasal immunization with $\mathrm{rLP} 4 / \mathrm{rLP} 6 / \mathrm{UspA} 2$ proteins combined with aqueous formulation of RC529. Vaccine 22: 3449-3456.

97. Shurin PA, Pelton SI, Tager IB, Kasper DL (1980) Bactericidal antibody and susceptibility to otitis media caused by nontypable strains of Haemophilus influenzae. J Pediatr 97: 364-369.

98. Faden H, Bernstein J, Brodsky L, Stanievich J, Krystofik D, et al. (1989) Otitis media in children. I. The systemic immune response to nontypable Haemophilus influenzae. J Infect Dis 160: 999-1004. 